\title{
Augmenting the Structures in a Swirling Flame via Diffusive Injection
}

\section{Jonathan Lewis, Agustin Valera-Medina, Richard Marsh, and Steven Morris}

Cardiff School of Engineering, Queen's Buildings, Cardiff CF24 3AA, UK

Correspondence should be addressed to Jonathan Lewis; lewisj19@cf.ac.uk

Received 17 February 2014; Revised 27 April 2014; Accepted 4 June 2014; Published 13 August 2014

Academic Editor: Kalyan Annamalai

Copyright ( 2014 Jonathan Lewis et al. This is an open access article distributed under the Creative Commons Attribution License, which permits unrestricted use, distribution, and reproduction in any medium, provided the original work is properly cited.

\begin{abstract}
Small scale experimentation using particle image velocimetry investigated the effect of the diffusive injection of methane, air, and carbon dioxide on the coherent structures in a swirling flame. The interaction between the high momentum flow region (HMFR) and central recirculation zone (CRZ) of the flame is a potential cause of combustion induced vortex breakdown (CIVB) and occurs when the HMFR squeezes the CRZ, resulting in upstream propagation. The diffusive introduction of methane or carbon dioxide through a central injector increased the size and velocity of the CRZ relative to the HMFR whilst maintaining flame stability, reducing the likelihood of CIVB occurring. The diffusive injection of air had an opposing effect, reducing the size and velocity of the CRZ prior to eradicating it completely. This would also prevent combustion induced vortex breakdown CIVB occurring as a CRZ is fundamental to the process; however, without recirculation it would create an inherently unstable flame.
\end{abstract}

\section{Introduction}

The depletion of fossil fuels and concern about the climate change have led to the development of new technologies to meet power generation demand, whilst maintaining security of supply and decreasing the environmental impact. The use of gas turbines, a well-developed technology, fired on nontraditional fuels, is an increasingly viable method for producing energy in the short to medium term. Fuels that can be used for this purpose range from those based on highly enriched hydrogenated blends to those that are produced from biomaterials [1-4]. Therefore, gas turbine technologies are evolving to cope with the use of these new fuels. However, operators are still finding problems with fuels that vary in composition, posing a new challenge to manufacturers to produce equipment with less stringent fuel requirements [5].

In order to reduce the emission of nitrogen oxides $\left(\mathrm{NO}_{x}\right)$, gas turbines operate using lean premixed combustion, utilising swirl stabilisation and resulting in the central recirculation zone (CRZ) of the flame becoming crucial. The CRZ provides heat to fresh reactants and anchors the flame. However, unless its size and shape are controlled, stability problems can arise. The CRZ can for instance readily extend back into the burner surrounding the fuel injector and facilitating early flashback (low stability limit) [6-8]. Flashback can be caused by (i) boundary layer flame propagation, (ii) turbulent flame propagation in the core flow, (iii) thermoacoustics, and (iv) upstream flame propagation of coherent vortical structures [7, 9-11].

Two of these mechanisms, that is, boundary layer flame propagation and upstream propagation of coherent structures, have been studied by several groups using natural gas. However, the use of unconventional fuels can be extremely detrimental to the control of these phenomena, and very little literature is available on this subject. High turbulence levels, one of the very useful features of swirling flow because of mixing potential, affect flashback limits detrimentally due to effects on turbulent flame speed $\left(S_{t}\right)$ and it has been found $[12,13]$ that the current theoretical approximations of $S_{t}$ do not agree with experimental values. Literature on this topic becomes more complex in terms of numerical modelling, but experiments tend to be different from numerical findings especially when complex flows are added to the field [13]. For instance, very little has been documented in terms of boundary layer propagation using atmospheric conditions $[3,14,15]$, and these findings only show the evolution of $2 \mathrm{D}$ 
structures without swirl and under atmospheric conditions. Thus, the current knowledge on these mechanisms cannot adequately describe the flashback propensity of most practical combustor designs. Therefore, the recognition of the real pattern of this phenomenon is crucial in order to have systems and models capable of utilising new alternative fuels.

Some authors [16-21] have observed that the CRZ has a close connection to the stability of the system, with its shape, strength, and curvature being of high importance to its resistance to flashback and blow-off [20, 22]. Regular precession occurs in the CRZ, with its appearance dependent on the heat transfer regime, the mode of injection, and the increase in the interaction of the hot products and fresh reactants when confinement is imposed. The CRZ behaves as an intermittent structure that will propagate downstream in order to release some internal pressure as a product of the confinement, and intense recirculation at moderate to high swirl numbers [20]. This intermittency can also be detrimental to the phenomenon of flashback as the CRZ will evolve into combustion induced vortex breakdown(CIVB) [23], boundary layer propagation [15], or the production of turbulent burning along the vortical axis [24], all of which can be damaging to the system.

Dam et al. [12] demonstrated the combustion induced breakdown of vortices as being a result of the high velocity zones of a flame squeezing the recirculation zone, causing the CRZ to propagate upstream, ultimately inhibiting the recirculation from occurring. I could be argued that CIVB also plays its part in boundary layer and turbulent flame propagation by augmenting the position of the flame in a way that makes them happen more readily [25].

In terms of unconventional fuels, the primary goal of introducing $\mathrm{CO}_{2}$ into the gas turbine combustor is to reduce the emissions of $\mathrm{NO}_{x}$. This is achieved by cooling the flame; thus, the Zeldovich mechanism can be reduced [26]. Previous experimental and numerical studies have investigated the effect of dilution of Syngas fuels with various additives, including carbon dioxide, nitrogen, and steam [27-31]. In the majority, these studies focus on fundamental characteristics of the combustion process. However, the work by Lee et al. [27] and Khalil et al. [1] actually investigated the effect of diluting the premix fuel had on the emission of $\mathrm{NO}_{x}$ and $\mathrm{CO}$ from a model gas turbine.

Lee et al. [27] showed that reduction in ppmv $\mathrm{NO}_{x}$ per unit power is logarithmically related to the heat capacity of the total diluent added. Since carbon dioxide has a higher heat capacity than steam or nitrogen, a smaller mass flow rate is required for a comparable reduction in $\mathrm{NO}_{x}$. Moreover, the use of $\mathrm{CO}_{2}$ from carbon capture and storage facilities could reduce costs as well as capture equipment further downstream the combustion zone.

This study focuses on, but is certainly not limited to, flames that require a pilot to maintain stability. The injection of $\mathrm{CO}_{2}$ is expected to augment the size and intensity of coherent structures whilst increasing mass flow rate through the exit nozzle, in much the same way methane pilot does, in such a way that CIVB is inhibited. The effect that the diffusive injection of air on stability will also be investigated and compared.

\section{Experimental Facilities}

A swirl burner constructed from stainless steel was used to examine the flame structure at atmospheric conditions (1bar, 293 K) at Cardiff University's Gas Turbine Research Centre (GTRC). External and sectioned views of the generic burner are presented in Figure 1. Secondary, full scale tests were performed using the high pressure combustion rig (HPCR), fitted with a proprietary gas turbine combustor, also at the GTRC. The rig is capable of delivering $5 \mathrm{~kg} \cdot \mathrm{s}^{-1}$ of air at $900 \mathrm{~K}$ and 16 barA, thus allowing combustors to be operated at conditions applicable to use in a power generation derivative gas turbine engine.

2.1. Generic Swirl Burner. A single tangential inlet (a) feeds the premixed air and fuel to an outer plenum chamber (b) which uniformly distributes the gas to the slot type radial tangential inlets (c) which impart the swirling momentum on the premixed flow. Swirling premixed air and fuel then pass into the swirl chamber (d) and then into the exit nozzle (e). The central diffusion fuel injector (f), through which nonpremixed gases are introduced to the combustion zone, extends centrally through the combustor body to the exhaust.

The geometric swirl number $\left(S_{g}\right)$ describes inlet conditions and burner geometry, allowing variations in flow pressure to be neglected [32]. It is defined in (1) for isothermal conditions, where density is constant, in radial type burner:

$$
S_{g}=\frac{A_{e} \cdot r_{t}}{A_{t} \cdot r_{e}} \cdot\left(\frac{Q_{t a}}{Q_{t o}}\right)^{2},
$$

where $A_{e}$ is the area of burner nozzle exit $\left(\mathrm{m}^{2}\right), r_{t}$ is the effective radius of tangential inlets (c) $(\mathrm{m}), A_{t}$ is the area of tangential inlet $\left(\mathrm{m}^{2}\right), r_{e}$ is the radius of burner nozzle exit $(\mathrm{m})$, $Q_{t a}$ is the tangential flow rate $\left(\mathrm{m}^{3} / \mathrm{s}\right)$, and $Q_{t o}$ is the total flow rate $\left(\mathrm{m}^{3} / \mathrm{s}\right)$.

During these trials the geometry was kept constant, so the only change in geometric swirl number was caused by the injection of gases through the pilot injector.

The system was fed using compressed air through flexible hoses and a Coriolis meter for flow rate metering. Bottled methane was used as main fuel at a constant flow rate during the trials, fed through flexible hoses passing through another Coriolis meter. A Dantec PIV (particle image velocimetry) system consisting of a dual cavity Nd: YAG Litron Laser of $532 \mathrm{~nm}$ capable of operating at $15 \mathrm{~Hz}$, a Dantec Dynamics laser sheet optics (9080X0651), was used to convert the laser beam into a $1 \mathrm{~mm}$ thick sheet. The laser sheet and thus the plane of measurement intersected the central axis of the burner exit nozzle. To record the images a Hi Sense MkII Camera model C8484-52-05CP was used, with 1.3 MPixel resolution at 8 bits. A $60 \mathrm{~mm}$ Nikon lens was used for resolution purposes, which allowed a field of view of approximately $75 \times$ $75 \mathrm{~mm}$, with a resolution of 5.35 pixels per $\mathrm{mm}$ and a depth of view of $1.5 \mathrm{~mm}$. The inlet air was seeded with aluminum oxide $\left(\mathrm{Al}_{2} \mathrm{O}_{3}\right)$ by an accumulator positioned $2.0 \mathrm{~m}$ upstream of the burner inlets.

After acquisition of the PIV data, a frame-to-frame adaptive correlation technique was then carried out with 


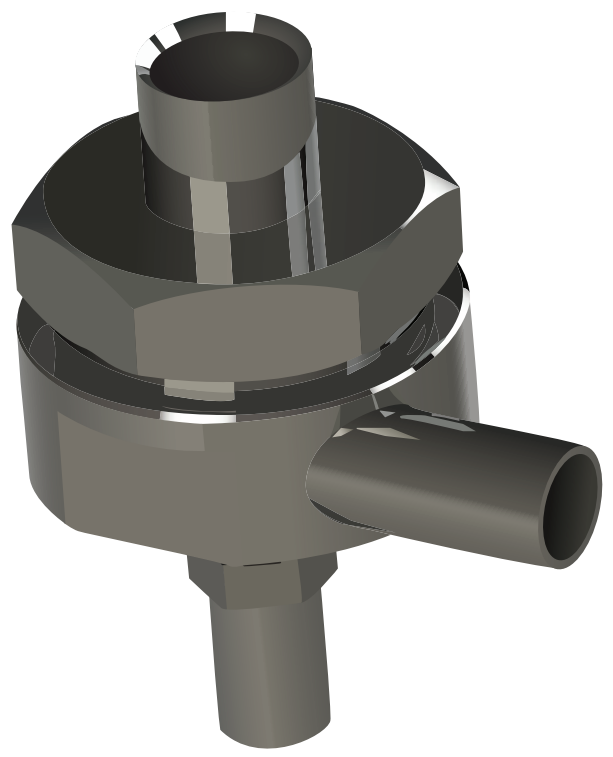

(a)

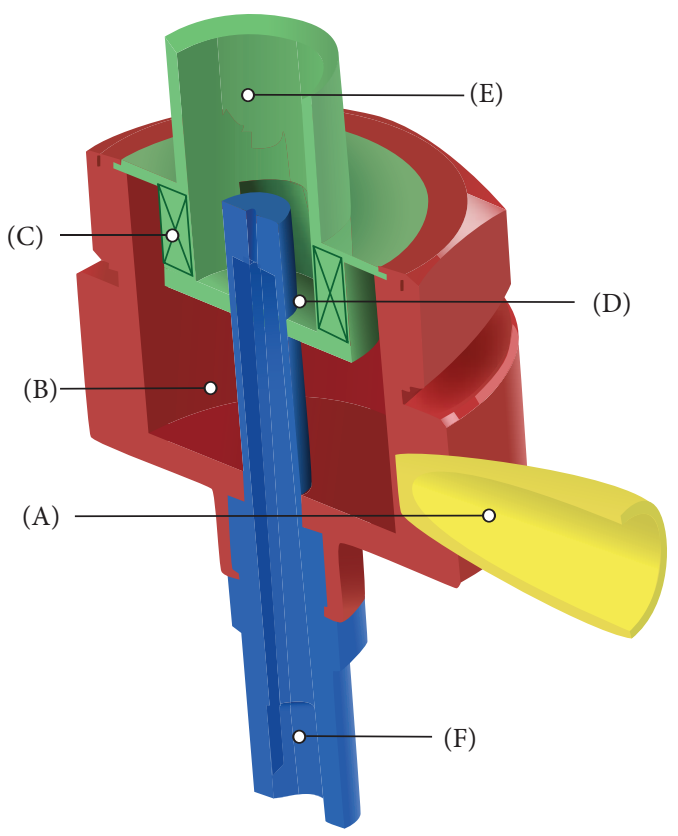

(b)

FIgURE 1: (a) External view and (b) sectioned schematic of the generic burner.

a minimum interrogation area of $32 \times 32$ pixels and a maximum of $64 \times 64$, with an adaptivity to particle density and velocity gradients. 150 pairs of frames per plane were used to create an average velocity map. Adaptivity in the analysis allowed very coherent images, with just a mask refinement of $\sim 15-20 \%$ of the entire field of view. In order to reduce the parallax error, the line of view of the camera was positioned exactly in the middle of the nozzle using a calibration grid provided by the system manufacturer. The grid was used to correct, via software, any positioning issue. The field of view was calibrated with the central line of the burner in the centre of the grid, thus ensuring that the position of the system would not affect the results.

The cross sectional area and velocities $(U)$ of the structures within the flame were assessed using the exported, numeric PIV data, with different components of the flame designated based on the axial velocity component, as indicated in Figure 2. These designations were assigned during previous experimentation with the generic burner [25]. A maximum value of $U_{a}$ (axial velocity) $=-0.3 \mathrm{~m} / \mathrm{s}$ was used to define the CRZ, removing any outlying areas of recirculation. A boundary of $U_{t}$ (total velocity) $=3 \mathrm{~m} / \mathrm{s}$ was used to define the HMFR, and this was the maximum value within of $U_{r}$ within the CRZ, as such flow with a velocity exceeding $3 \mathrm{~m} / \mathrm{s}$ had a momentum higher than that in CRZ.

2.2. Method of Initial Experimentation. Varying amounts of carbon dioxide, air, and methane were injected through the diffusive pilot of the AGSB to assess the effect on flame structure. The three gases were selected for their differing combustion properties:
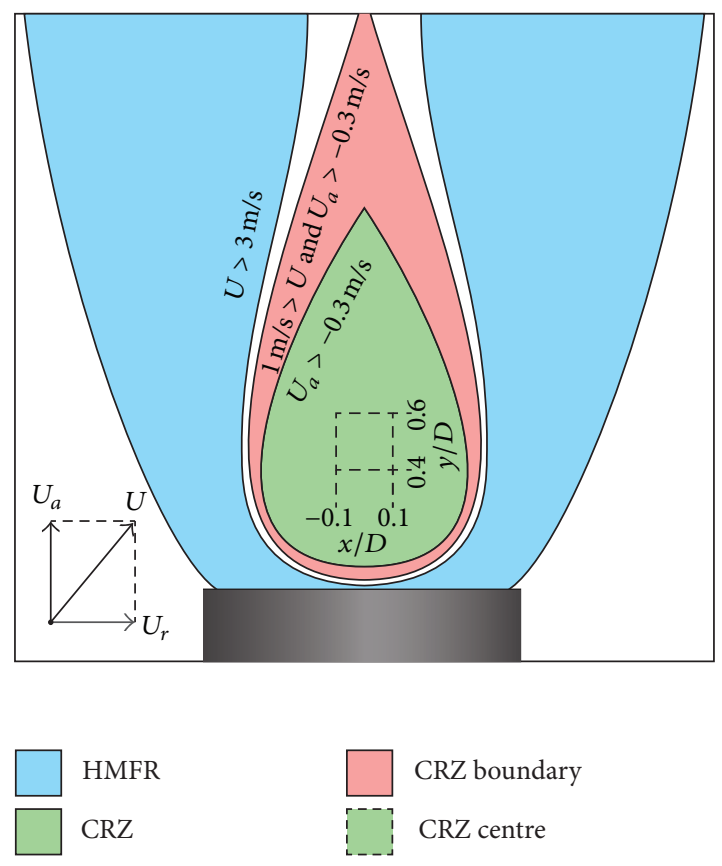

FIGURE 2: Designation of areas within the flame.

(i) methane which is often used as a pilot fuel in gas turbines and will increase the global equivalence ratio of the flame, global equivalence ratio being that of the premixed air and methane, and the

(ii) carbon dioxide which does not affect oxidant-to-fuel ratio but has been shown in previous studies to alter flame conditions [27-31], 
TABLE 1: Details of AGSB test points 1-26.

\begin{tabular}{|c|c|c|c|c|c|c|c|c|c|}
\hline & \multicolumn{4}{|c|}{ Flow rates $(\mathrm{g} / \mathrm{s})$} & \multirow[b]{2}{*}{$\begin{array}{l}\text { Total power } \\
(\mathrm{kW})\end{array}$} & \multirow[b]{2}{*}{ Isothermal $S_{g}$} & \multirow{2}{*}{$\begin{array}{l}\text { Bulk exit velocity } \\
(\mathrm{m} / \mathrm{s})\end{array}$} & \multirow[b]{2}{*}{$\operatorname{Re}$} & \multirow[b]{2}{*}{$\begin{array}{r}\mathrm{AFT} \\
(\mathrm{C})\end{array}$} \\
\hline & Test point & $\begin{array}{c}\text { Pilot } \\
\text { (through f) }\end{array}$ & total & $\Phi$ & & & & & \\
\hline \multirow{8}{*}{ Methane } & 1 & 0.000 & 3.640 & 1.00 & 10.0 & 1.05 & 4.88 & 4361 & 1953 \\
\hline & 2 & 0.015 & 3.655 & 1.07 & 10.7 & 1.04 & 4.90 & 4379 & 1928 \\
\hline & 3 & 0.030 & 3.670 & 1.15 & 11.5 & 1.02 & 4.92 & 4397 & 1875 \\
\hline & 4 & 0.045 & 3.685 & 1.22 & 12.2 & 1.01 & 4.94 & 4415 & 1816 \\
\hline & 5 & 0.059 & 3.699 & 1.30 & 13.0 & 0.99 & 4.96 & 4433 & 1760 \\
\hline & 6 & 0.074 & 3.714 & 1.37 & 13.7 & 0.98 & 4.98 & 4451 & 1702 \\
\hline & 7 & 0.089 & 3.729 & 1.45 & 14.5 & 0.97 & 5.00 & 4468 & 1645 \\
\hline & 8 & 0.104 & 3.744 & 1.52 & 15.2 & 0.95 & 5.02 & 4486 & 1589 \\
\hline \multirow{6}{*}{ Air } & 9 & 0.000 & 3.640 & 1.00 & 10.0 & 1.05 & 4.88 & 4361 & 1953 \\
\hline & 10 & 0.100 & 3.740 & 0.97 & 10.0 & 1.00 & 5.02 & 4482 & 1924 \\
\hline & 11 & 0.201 & 3.841 & 0.94 & 10.0 & 0.95 & 5.15 & 4602 & 1902 \\
\hline & 12 & 0.301 & 3.941 & 0.92 & 10.0 & 0.90 & 5.29 & 4722 & 1875 \\
\hline & 13 & 0.402 & 4.042 & 0.90 & 10.0 & 0.86 & 5.42 & 4843 & 1846 \\
\hline & 14 & 0.502 & 4.142 & 0.87 & 10.0 & 0.82 & 5.56 & 4963 & 1817 \\
\hline \multirow{8}{*}{ Carbon dioxide } & 15 & 0.000 & 3.640 & 1.00 & 10.0 & 1.05 & 4.88 & 4361 & 1953 \\
\hline & 16 & 0.025 & 3.665 & 1.00 & 10.0 & 1.04 & 4.92 & 4391 & 1931 \\
\hline & 17 & 0.050 & 3.690 & 1.00 & 10.0 & 1.03 & 4.95 & 4421 & 1923 \\
\hline & 18 & 0.074 & 3.714 & 1.00 & 10.0 & 1.03 & 4.98 & 4451 & 1914 \\
\hline & 19 & 0.099 & 3.739 & 1.00 & 10.0 & 1.02 & 5.02 & 4481 & 1906 \\
\hline & 20 & 0.124 & 3.764 & 1.00 & 10.0 & 1.01 & 5.05 & 4510 & 1897 \\
\hline & 21 & 0.149 & 3.789 & 1.00 & 10.0 & 1.00 & 5.08 & 4540 & 1889 \\
\hline & 22 & 0.174 & 3.814 & 1.00 & 10.0 & 0.99 & 5.12 & 4570 & 1881 \\
\hline
\end{tabular}

(iii) air which will reduce the flames global equivalence ratio.

In order to allow comparison between results, premix flow rates of $0.2 \mathrm{~g} / \mathrm{s}$ methane and $3.44 \mathrm{~g} / \mathrm{s}$ air were kept constant; these equate to a $10 \mathrm{~kW}$, stoichiometric mixture. Details of the test points are displayed in Table 1. The flames are unconfined, as such ambient air will be entrained, and they will actually be leaner than the defined equivalence ratio.

The experimental method was the same for all three gases: the flame was lit and 150 images were recorded with the PIV system, and the flow rate of diffusion gases was then increased in regular intervals, with a further 150 images recorded at each interval, until the flame reached a point of quasistability. In order to maintain inlet and outlet conditions between test points, the flame was left alight.

\section{Results}

The PIV results in Figure 3 demonstrate the effect of introducing methane through the pilot on the flame structure. The left hand side of the PIV images shows the axial velocity $\left(U_{a}\right)$ of the flows on scale of -5 to 8 meters per second. Negative velocities indicate that the flow downstream, towards the burner exit. On the right hand side of images total velocity $\left(U_{t}\right)$ is shown, on a scale of 0 to 8 meters per second. The total velocity is a measure of magnitude, regardless of direction, so no negative velocities are possible. The combination of image masking and the neighbourhood validation causes the data shown to underestimate the velocity of the fluid as it leaves the exit nozzle. This is why the fluid appears to have exceptionally low velocities in the region where $0<y / D<0.1$.

The flow in test point (TP) 1 has the highest velocities at $0.25 y / D$ and $\pm 0.55 x / D$, indicated by the red regions in both scalar plots, and there is also a subregion where axial velocity exceeds the $8 \mathrm{~m} / \mathrm{s}$ on the scale shown. The unburned, premixed reactants are at their greatest velocity near the outside of the exit nozzle, where the tangential momentum imparted by the radial swirl passages has forced the flow away from the burner axis but boundary effects are negligible. The centrifugal force on the fluid also means that the reactants are the densest at the inner wall of the exit nozzle. Combustion occurs when the reactants leaving the exit nozzle, a significant increase in axial velocity results from hot gas expansion. An increase in radial velocity also occurs. The combined tangential and axial momentum of the products results in flow spreading in the $x$ and $y$ directions on the plane shown. As spreading occurs and the combustion process is complete, velocities decrease. Tangential momentum results in predominant expansion in the $x$ direction being away from the burner axis. This creates a low pressure zone along the vertical burner axis, with a pressure differential that causes combustion products to flow towards the central axis 

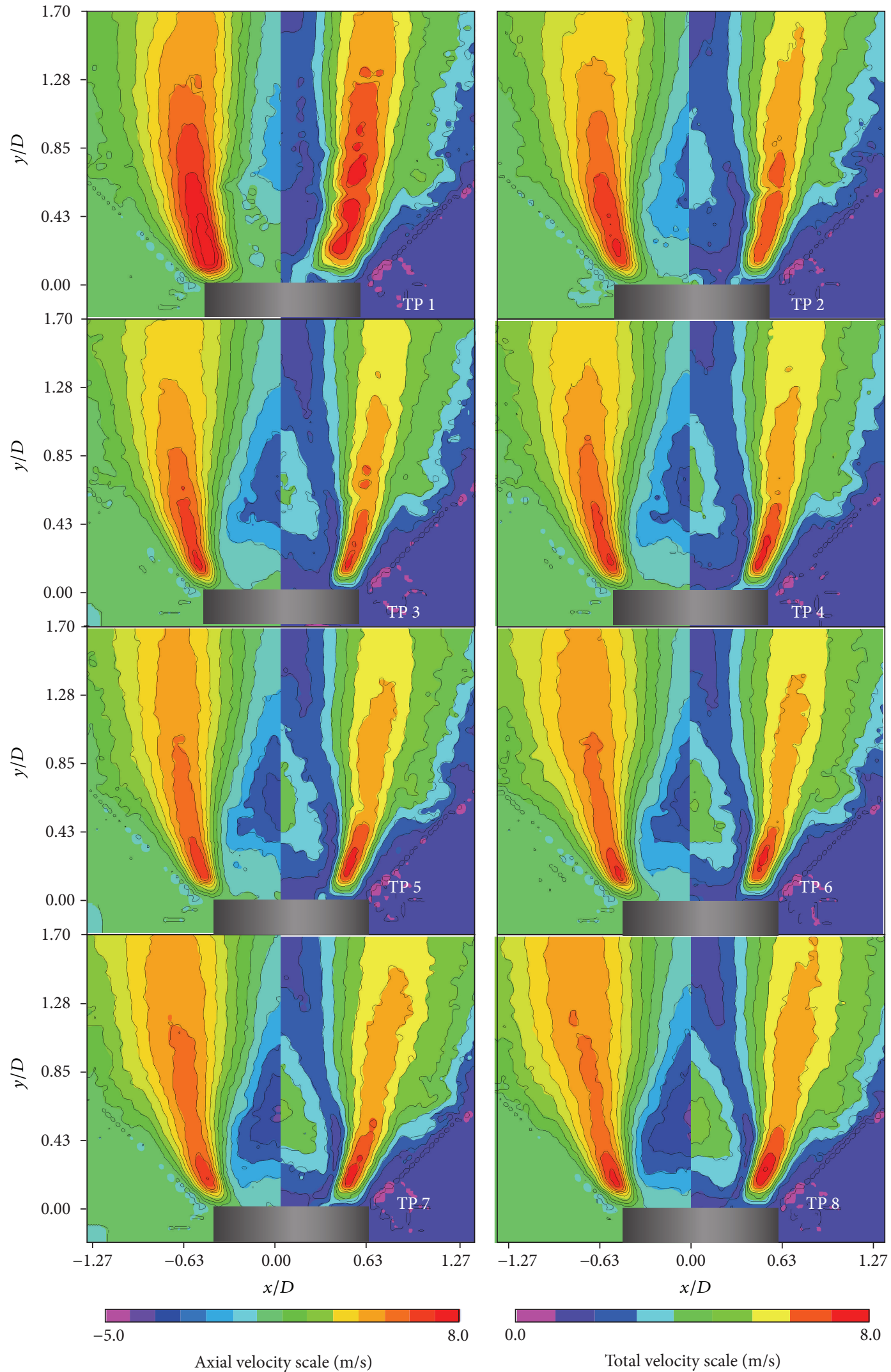

FIGURE 3: Scalar plots of TP 1 to TP 8 in the AGSB as detailed in Table 1, the left half of each image indicates axial velocity, and the right half total velocity. 
and burner exit. The stagnation of the flow prior to being recirculated is indicated by the turquoise region in the axial velocity profile.

TP 1 has a very narrow, low velocity, CRZ. Within the recirculation zone the maximum value of $U_{t}$ is $2 \mathrm{~m} / \mathrm{s}$ and the minimum value of $U_{a}$ is $-1 \mathrm{~m} / \mathrm{s}$. The axial velocity of the recirculated gases becomes positive at $y / D=0.4$. The introduction of methane through the pilot results in an increase in size and velocity of the CRZ, which can be seen in TP 2. The shape of recirculation zone also changes, with the width increasing disproportionally at its base. The negative axial velocity of the recirculated gases at the centre of the CRZ increases significantly, exceeding $2 \mathrm{~m} / \mathrm{s}$. Due to the increase in velocity of the recirculated gases, the region of negative axial velocity extends toward the burner exit, as a result the CRZ becomes elongated. The velocity in the HMFR in TP 2 is significantly lower than that in TP 1, in terms of both axial and total velocity; it appears to have become narrower as a result of the CRZ expanding.

The alteration in shape becomes further exaggerated as the flow rate of pilot methane increases through test points 3 to 8 . With each increase in the flow rate of diffusive methane the width of the reverse flow region increases, as do the negative axial and total velocities of the gases within them. The injection of methane reduces the peak velocity of the reacting flows in the HMFR; however, the shape and overall velocity profiles in these regions remain largely unaffected.

The diffusive injection of air has a markedly different effect on the flow structure than was observed with methane. PIV images in Figure 4 effectively demonstrate how the air causes the size and mean velocity of the CRZ to reduce, eventually leading to its complete destruction. Test points 9 through 12 are displayed as in Figure 3, the left hand side of the images showing axial velocity $\left(U_{a}\right)$ and the right hand side of images total velocity $\left(U_{t}\right)$. The full, axial, and total velocity profiles of test points 13 and 14 are shown due to the high level of asymmetry in the flow structure.

The flow structure and velocities of TP 9 are very similar to those observed in TP 1 ; this is to be expected as the premixed flow rates are the same. The structure of TP 10 however is very different to that of TP 2. Rather than increasing with diffusive flow rate, the width of the recirculation zone is unaltered from TP 9. Although total velocity is also unaltered, negative axial velocity is actually reduced.

The total and axial velocities in the HMFR of TP 11 are considerably lower than in TPs 9 and 10. The flame is also displaying significant asymmetry, with the low velocity recirculation zone positioned left of of the central axis of the burner. The increased addition of air continues to reduce the velocity of the flow within the CRZ and the HMFR in TP 12, whilst the asymmetry becomes more exagerated. The diffusive flow rate in TP 13 has caused an almost complete reduction of the $\mathrm{CRZ}$, although a region exists where axial velocities are just below 0 . In TP 14 the CRZ no longer exists, with no flow in the negative axial direction. Instead the CRZ is replaced by a central region of high positive axial velocity, on the right of the central burner axis as shown. The likely cause of the CRZ destruction is combustion inside of the CRZ, causing an expansion of gases along the central axis of the burner. This gradually reduces the pressure differential that is required in order to induce recirculation.

The effect of reduced swirl number may also contribute to the results seen in test points 13 and 14, where the $S_{g}$ is lower than that observed with $\mathrm{CO}_{2}$ or $\mathrm{CH}_{4}$. The delivery system restricted the flow rate of $\mathrm{CO}_{2}$ and $\mathrm{CH}_{4}$ to the values shown in Table 1. However, since the relationships between diffusive flow rate, for all three gases, and structure augmentation were established for geometric swirl numbers greater than 1, the effects of swirl number are considered secondary.

Carbon dioxide has a very similar effect of flow structure to methane; as a result, the progression caused by its diffusive injection between TP 15 to TP 22, which is shown in Figure 5, resembles the progression in Figure 3.

Test points 15 through 22 are displayed in Figure 3, the left hand side of the images showing axial velocity $\left(U_{a}\right)$ and the right hand side of images total velocity $\left(U_{t}\right)$. The flow structure of TP 15 resembles those of TP 1 and TP 9 where a narrow and low velocity recirculation zone exists. However, over the measured plane, the velocities of the flows in TP 15 are lower than those observed in TPs 1 and 9. This is likely the result of slightly different initial flow rates, which can be caused by changes in ambient conditions or compressor pressure. As the proceeding alterations in flow structure are of interest, this inconsistency in initial condition is acceptable.

In TP 16 it can be seen that the CRZ increases in width as carbon dioxide is diffusively injected, and the total and axial velocities within the CRZ are also increased. With methane this increase in velocity coincided with an elongation of the recirculation, with the region of reverse flow approaching the burner exit; with carbon dioxide this was not observed. A reduction in velocity in the HMFR is evident between TP 15 and TP 16.

Between TP 16 and TP 22, the increase in diffusive $\mathrm{CO}_{2}$ flow rate results in an increase in the size of the CRZ, and axial and total velocities within it also increase, with the reverse flow region propagating slightly upstream. This reduction is expected as the dilution effect of the $\mathrm{CO}_{2}$ will reduce flame temperatures and the expansion of the gas. The observed reduction in axial velocity is less pronounced than the reduction in total velocity, which suggests that radial velocity is reduced significantly.

3.1. Effect on Turbulence. When $\mathrm{CO}_{2}$ is diffusively injected, the low velocity boundary that exists between the CRZ and the HMFR appears to be wider when $\mathrm{CH}_{4}$ is injected. This is particularly evident when comparing vector plots of TP 5 , with methane injection and TP 19, with carbon dioxide injection, which are shown in Figure 6. The CRZ is also visibly less defined in TP 19 than in TP 5; this is the result of higher levels of turbulence within the recirculation zone.

When all gases entering the burner are introduced tangentially, they are considered to be fully mixed prior to entering the combustion zone. With diffusive injection, mixing is forced to occur in the region of the burner exit, resulting in unequal mixtures within the combustion zone.

Simulation with CHEMKIN using the GRIMech 3.0 mechanism [33] predicts that under laminar conditions the 


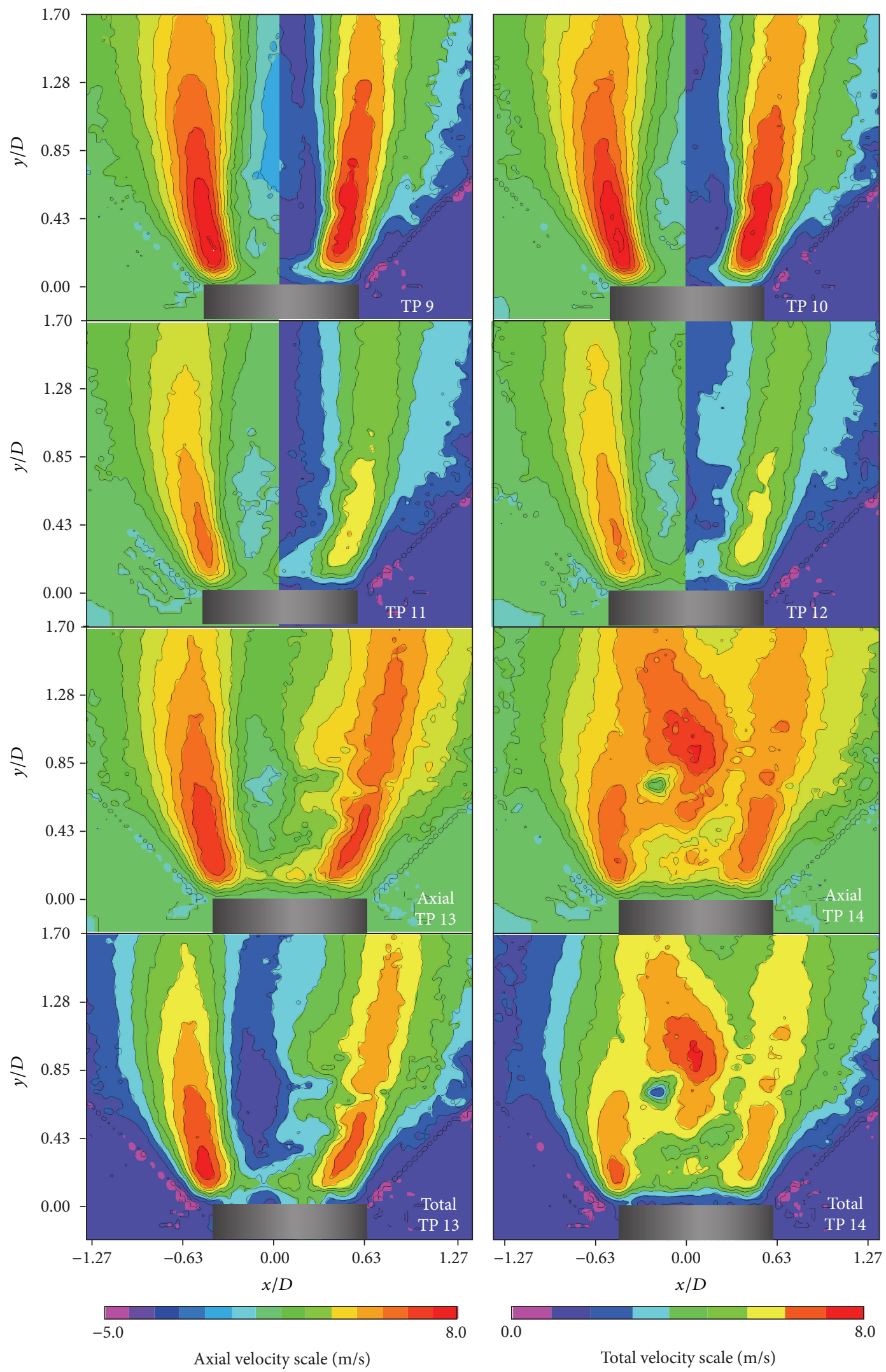

FIGURE 4: Scalar plots of TP 9 to TP 14 in the AGSB as detailed in Table 1, TP 9 to TP 12 the left half of each image indicates axial velocity, and the right half total velocity. TP 13 and TP 14 show full frames of axial and total velocity. 

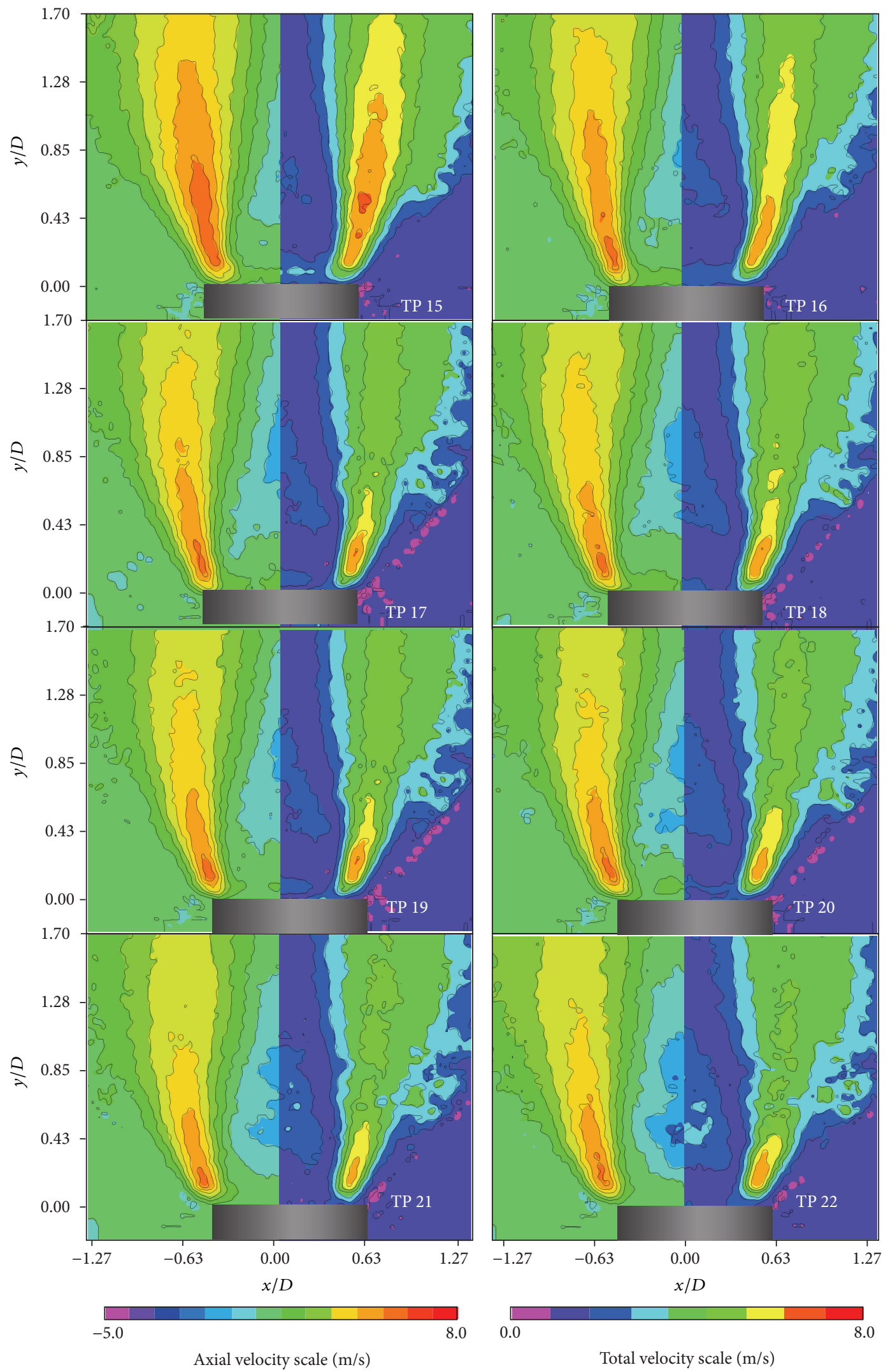

FIGURE 5: Scalar plots of TP 15 to TP 22 in the AGSB as detailed in Table 1, the left half of each image indicates axial velocity, and the right half total velocity. 


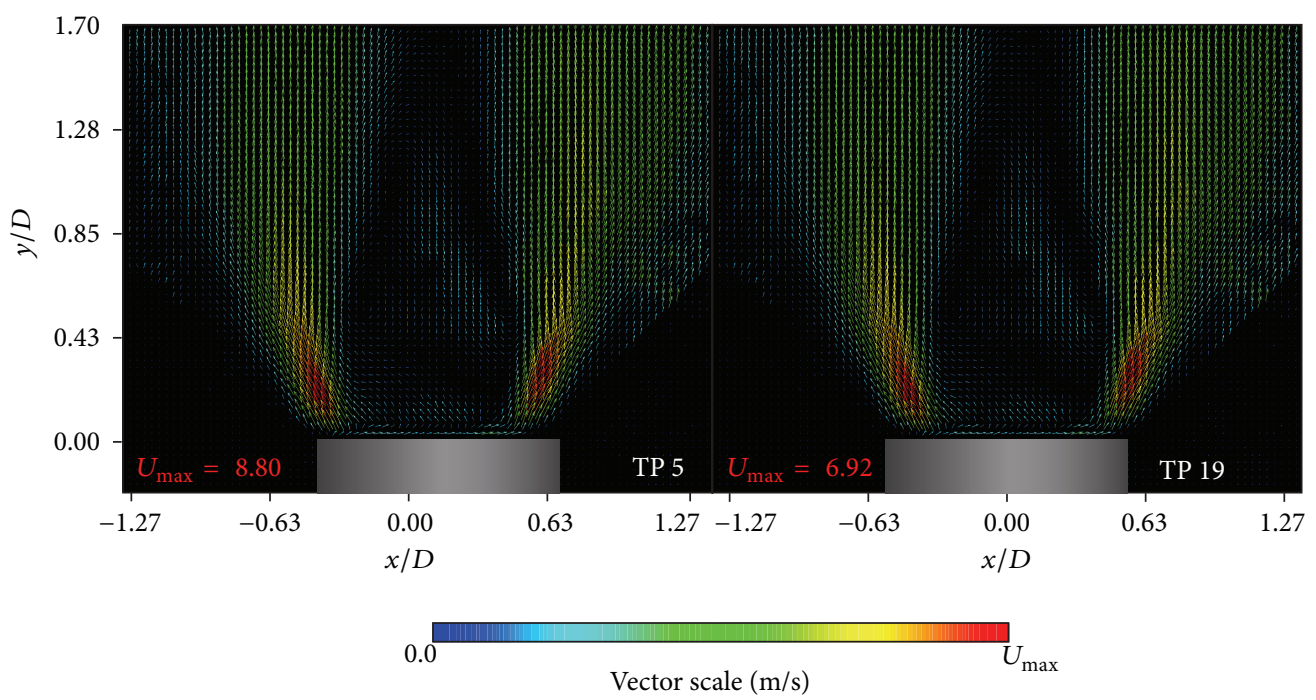

FIgURE 6: Vector plots of TP 5 and TP 19 in the AGSB with altered premix air flow rate and pilot $\mathrm{CO}_{2}$ flow rate as detailed in Table 1: vectors indicate velocity and direction.

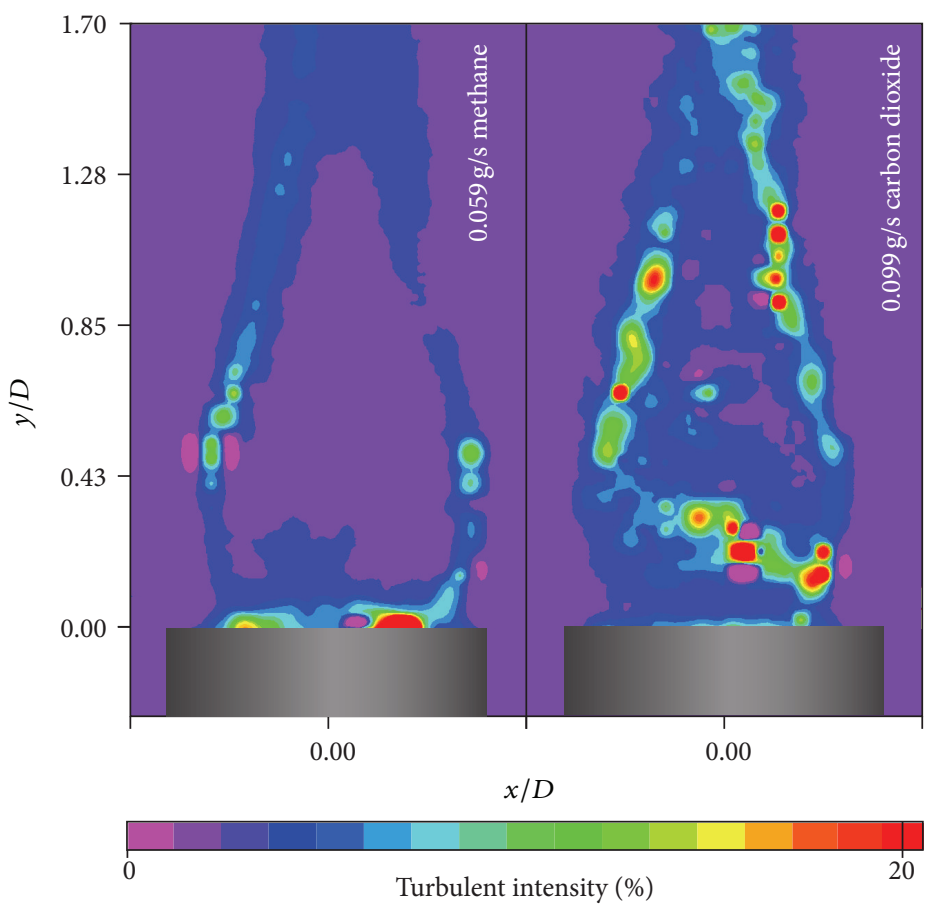

FIgURE 7: Comparison of turbulent intensity between flames in TP 5 and TP 19.

peak methane burning rate occurs at a rich equivalence ratio of approximately 1.1. Previous work has also utilised the combination of CHEMKIN and GRIMech 3.0 to show how $\mathrm{CO}_{2}$ dilution reduces laminar burning rates [34]. With premixed gaseous fuel, combustion normally occurs at the boundary between the CRZ and the HMFR, where fresh reactants and hot products combine. The amalgamation of delayed mixing and unequal alterations in burning rates affect the ability of the flame to propagate toward CRZ when $\mathrm{CO}_{2}$ and $\mathrm{CH}_{4}$ are injected.

The increase is in both size and mean velocity caused by $\mathrm{CO}_{2}$ injection are not as prominent as with methane, and the CRZ has a less well-defined shape as an irregular flame front develops at the boundary between it and the HMFR, as is reflected in the level of the turbulent intensity. Figure 7 represents the turbulent intensity of the flames in 


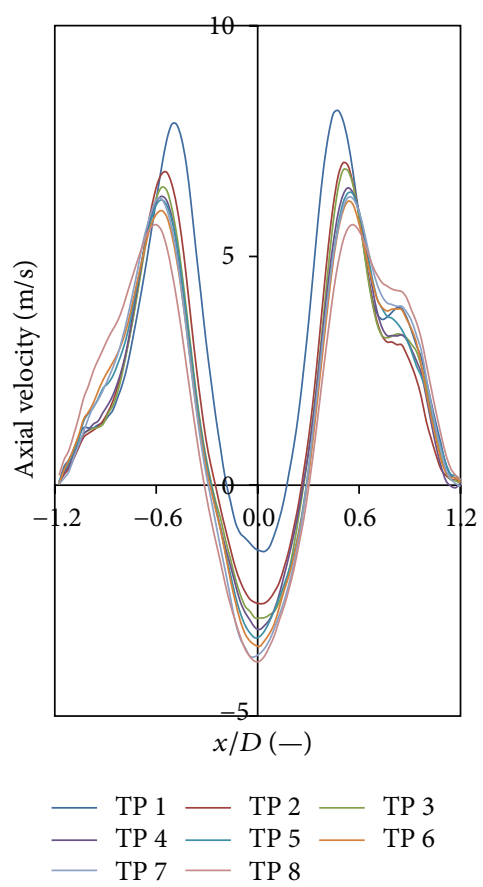

(a)

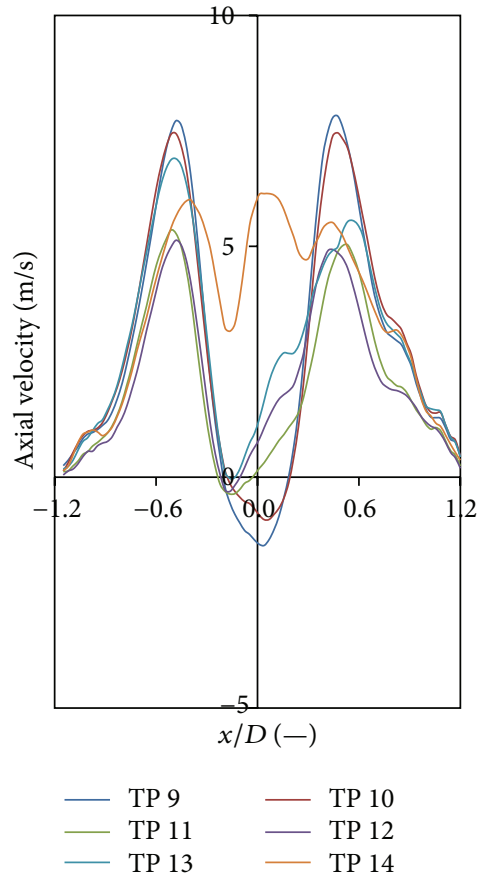

(b)

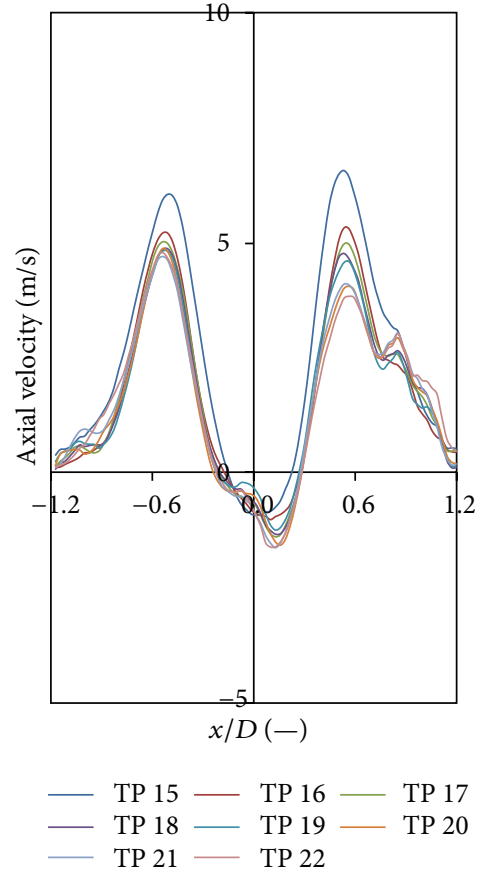

(c)

Figure 8: Axial velocities at $y / D=0.5$ for test points detailed in Table 1 with the diffusive injection of (a) methane, (b) air, and (c) carbon dioxide.

TP 5 and TP 19 in the axial-radial plane. The method for calculating turbulent intensity in the measured plane (I), at each measurement point in the PIV frame, is shown in (2) and (3):

$$
I=\frac{\sqrt{k} \mathrm{e}}{\sqrt{U_{a}^{2}+U_{r}^{2}}},
$$

where $k e$ is the turbulent kinetic energy $\left(\mathrm{m}^{2} / \mathrm{s}^{2}\right)$

$$
k e=\frac{1}{2} \cdot\left(u_{a}^{\prime 2}+u_{r}^{\prime 2}\right)
$$

And $u_{a}^{\prime}$ is the root-mean-square of turbulent velocity fluctuations in the axial direction $(\mathrm{m} / \mathrm{s}), u_{r}^{\prime}$ is the rootmean-square of turbulent velocity fluctuations in the radial direction $(\mathrm{m} / \mathrm{s}), U_{a}$ is the axial velocity $(\mathrm{m} / \mathrm{s})$, and $U_{r}$ is the radial velocity $(\mathrm{m} / \mathrm{s})$.

The flame with diffusive methane injection has a very low turbulent intensity CRZ $(\bar{I}=1.12 \%)$ and $\operatorname{HMFR}(\bar{I}=0.32 \%)$, with the intermediary boundary layer standing out due to the increased turbulent intensity $(\bar{I}=3.07 \%)$. The HMFR of the flame with diffusive carbon dioxide injection also has a very low turbulence level $(\bar{I}=0.44 \%)$. However, the turbulent intensity of the CRZ $(\bar{I}=2.93 \%)$ and the intermediate boundary layer $(\bar{I}=4.73 \%)$ are significantly higher.

The velocities in the methane injected flame are greater than those of the carbon dioxide injected flame, which has to be considered when comparing turbulent intensity, which is dependent on local velocity. When comparing the rootmean-square of the turbulent velocity fluctuations in the axial-radial plane, the mean turbulent kinetic energy levels in the CRZ boundaries of the methane and carbon dioxide flames are actually very similar, $\bar{k}=48.32 \mathrm{~m}^{2} / \mathrm{s}^{2}$ and $\bar{k}=$ $44.70 \mathrm{~m}^{2} / \mathrm{s}^{2}$, respectively, whereas the CRZ of the carbon dioxide injected flame displays significantly more turbulence $\left(\bar{k}=15.22 \mathrm{~m}^{2} / \mathrm{s}^{2}\right)$ than that of the methane flame $(\bar{k}=$ $\left.0.93 \mathrm{~m}^{2} / \mathrm{s}^{2}\right)$.

3.2. Effect on Momentum. The axial velocity profiles at $y / D=$ 0.5 for all test points are plotted in Figure 8, and a moving average of 5 data points is applied to smooth out local fluctuations from the global trend, for easier visual interpretation. In Figure 8(a), the profile of test point 1 demonstrates the negative axial velocities of the CRZ on the central axis of the burner, at $x / D=0$. The velocities increase either side of the burner axis, peaking at the centre of the HMFR before reducing again as the unconfined flow dissipates. The progressive introduction of methane produces incremental increases in the negative velocities in recirculation zone, whilst incrementally reducing the velocity within the high momentum flow region. The point at which the lines cross the axis also diverges from $x / D=0$, indicating that the CRZ is increasing in width whilst the HMFR is getting narrower. The profiles in Figure 8(b) demonstrate clearly how the introduction of air causes an asymmetrical distortion, and ultimate destruction of the CRZ. Between test point 9 and TP 10, the changes in velocity resemble those seen due to the injection of methane. Between TP 10 and TP 13, there is a significant reduction in the peak velocity in the right hand side of the HMFR. The CRZ in the positive radial 


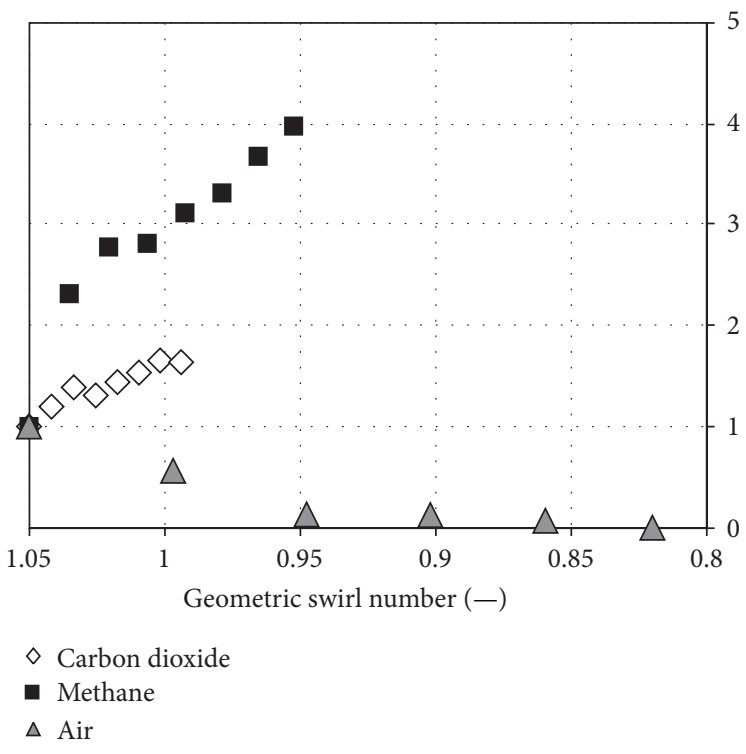

(a)

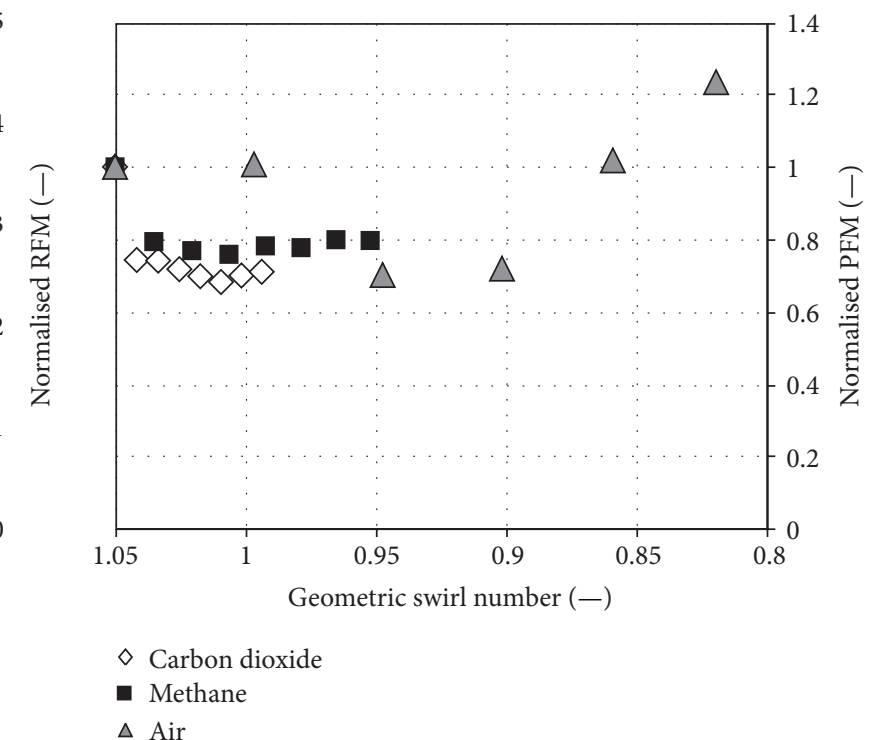

(b)

Figure 9: The (a) reverse and (b) positive flow momentum for all test points at $y / D=0.5$.

direction of the burner is also subject to a greater change than in the negative radial direction, with a large incremental increase in the axial velocity seen. Between test points 13 and 14 a dramatic change occurs, with the entire range of axial velocities becoming positive, and the peak velocity occurring at $x / D=0$.

However, the axial velocity profiles of the flames with $\mathrm{CO}_{2}$ injection, test points 15 to 22, display the same behaviour as observed with the injection of $\mathrm{CH}_{4}$. The diffusive injection causes the positive axial velocities in the HMFR to decrease, whilst the negative axial velocities in the CRZ increase. There is also evidence of the CRZ becoming wider as the value of $x / D$ when axial velocity which equals 0 increases with diffusive flow rate, although the expansion is not as prominent as observed with methane injection.

The amount of stabilisation provided by the CRZ can be assessed by the reverse flow momentum (RFM) of the gases within it. Momentum is a product of velocity and mass, as the temperature, and as a result, density of the gases within the CRZ and the reverse flow momentum are unknown. The volume of a particular measurement point is fixed; therefore, if variations in density are ignored, it is possible to compare the flow momentum by comparing velocity. As such, changes in reverse flow momentum (RFM) at $y / D=0$ are assessed by determining the areas below the $x$-axis, which are bound by the curves plotted in Figure 8.

The RFM of each test point is plotted against the isothermal geometric swirl number in Figure 9(a), with the results of TPs 1 to 8 normalised against the fully premixed flame of TP 1. Similarly, TPs 9 to 14 are normalised against TP 9, and TPs 15 to 22 are normalised against TP 15 .

The increased diffusive injection of methane, which reduces $S_{g}$ in accordance with (1), results in an increase in the RFM of the CRZ. Initially there is a very large increase, with the RFM of TP 22.3 times greater than TP 1, and RFM then increases linearly with diffusive flow rate. The injection of diffusive $\mathrm{CO}_{2}$ also increases the RFM of the CRZ; however, the rate of increase is significantly less than the one observed with $\mathrm{CH}_{4}$. Injection of air has an opposing effect, with RFM reducing as the amount of air injected increases. The effects on positive flow momentum (PFM), which are calculated in a similar fashion to RFM at $y / D=0$, with the area bound by the curve being positive of the $x$-axis, are shown in Figure 9(b). The injection of both $\mathrm{CO}_{2}$ and $\mathrm{CH}_{4}$, with initial diffusive flow rates causing $21 \%$ and $26 \%$ reductions in PFM, respectively. The increase in diffusive flow rate then has very little effect on the PFM, with the range of reduction of $\mathrm{CH}_{4}$ being $21-25 \%$ and the range of reduction of $\mathrm{CO}_{2}$ being $26-$ $32 \%$.

The complete alteration in structures caused by the introduction of air means that there are two effects on the PFM. The gradual destruction of the CRZ means that the main reaction zone expands, and the velocity in the HMFR reduces. Conversely, the destruction of the CRZ means that the regions of positive flow increases; the velocity in these regions will also increase due to the axial momentum of the injected air.

The radial velocity profiles at $y / D=0.5$ for all test points are plotted in Figure 10, again a moving average of 5 data points is applied. In Figure 10(a), the radial velocities of TPs 1 to 8 are shown. Rotational symmetry about the origin is expected with regard to radial velocity; however, due to the slightly asymmetry in the burner this is not seen, with TP 1 displaying entirely negative radial velocities within the CRZ. The increased injection of $\mathrm{CH}_{4}$ causes a general, incremental increase in radial velocity. TP 8 displays peak positive and negative values that are approximately as twice as those seen in TP 1. 


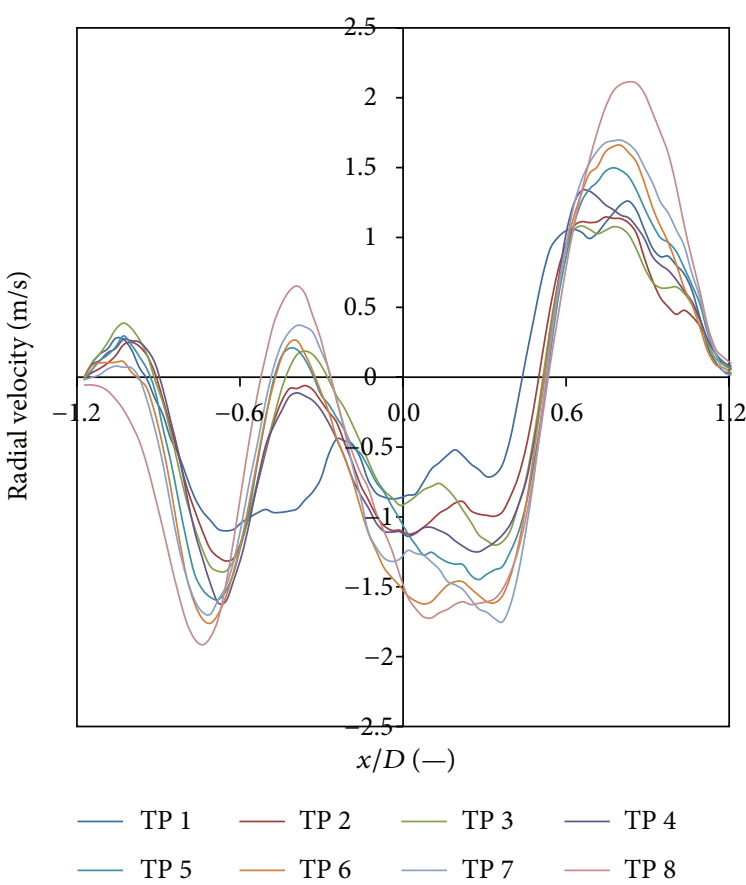

(a)

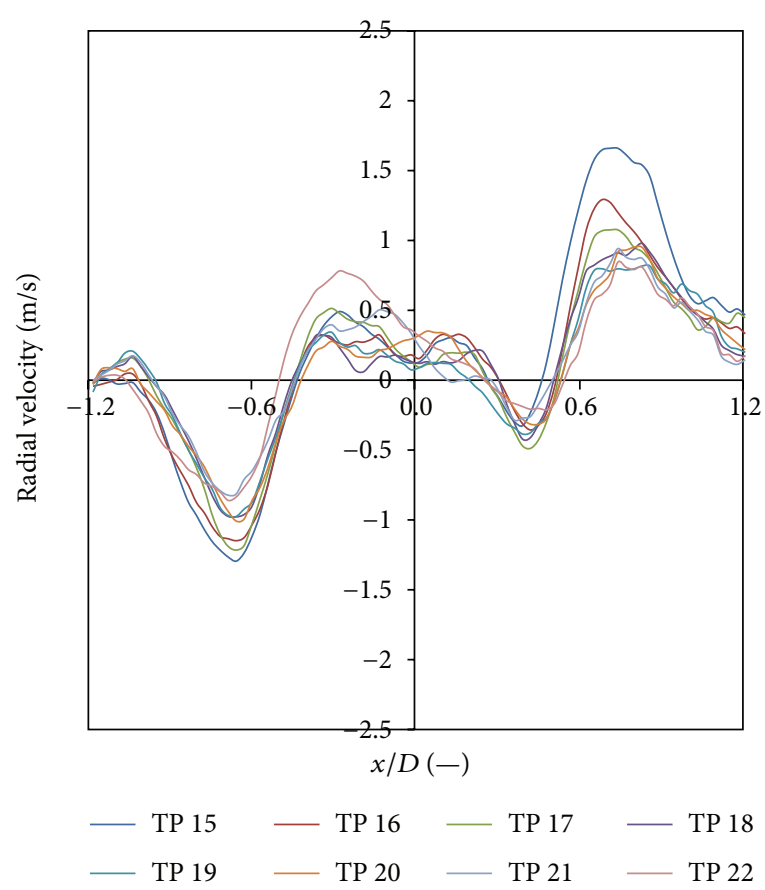

(b)

Figure 10: Radial velocities at $y / D=0.5$ for test points detailed in Table 1 with the diffusive injection of (a) methane and (b) carbon dioxide.

Coupled with the decrease in axial velocities these results suggest that the level of swirl has increased, which is why the RFM has increased relative to the PFM. The different effect is observed with the diffusive injection of $\mathrm{CO}_{2}$; the radial plots for test points 15 to 22 are shown in Figure 10(b). A slight change in flame structure means that velocities within the $\mathrm{CRZ}$ are all positive. The injection of $\mathrm{CO}_{2}$ has very little effect on the radial velocity inside the recirculation zone; however, the radial velocity in the HMFR decreases.

3.3. Effect on the CRZ. Figure 11 shows the effect on the size and velocity of the central recirculation zone, based on the corresponding geometric swirl number. As in Figure 8, the results are normalised against the fully premixed case to allow easier comparison. Figure 11(a) shows how both $\mathrm{CO}_{2}$ and $\mathrm{CH}_{4}$ injection cause the size of the $\mathrm{CRZ}$ to increase dramatically until reaching $\mathrm{Sg}=1.03$ for $\mathrm{CO}_{2}$ and 1.02 for $\mathrm{CH}_{4}$; the size then increases at a steady linear rate. The injection of $\mathrm{CH}_{4}$ has a greater effect on the size of the CRZ than $\mathrm{CO}_{2}$ does, with the increased velocity of the recirculated flow resulting in the wider CRZ base seen in Figure 3 than in Figure 5.

This increased velocity is demonstrated in Figures 11(b) and $11(\mathrm{c})$, where the mean velocity in the CRZ is in its entirety, and its central regions are calculated. Over the region as a whole, a steady linear increase, of a similar order, is observed with both $\mathrm{CO}_{2}$ and $\mathrm{CH}_{4}$, with all but one point falling on the same line. However, as the position and size of CRZ are calculated based on a component of the velocity, the mean velocity over the entire CRZ is influenced by the constraints applied. The central region, which is determined geometrically, is a better reflection on changes in velocity; a linear, sixfold increase is observed with methane, whereas $\mathrm{CO}_{2}$ only causes a threefold, nonlinear, increase.

3.4. Effect on the HMFR. Another structure in a swirling flame is the high momentum flow region; this is high velocity region which contains the main reaction zone of the flame. In this study, it is defined as being the region where axial velocity exceeds $3 \mathrm{~m} / \mathrm{s}$.

The effects of pilot injection on the size and mean velocity in this region are shown in Figure 12; it is observed that the effects of $\mathrm{CO}_{2}$ and $\mathrm{CH}_{4}$ injection differ. As increasing diffusive flow rates of both $\mathrm{CO}_{2}$ and $\mathrm{CH}_{4}$ cause $\mathrm{S}_{\mathrm{g}}$ to approach 1.02 , a reduction in cross sectional area of the HMFR is observed, below values of $S_{g}=1.02$, and $\mathrm{CO}_{2}$ continues to cause a reduction in HMFR area, whilst $\mathrm{CH}_{4}$ results in a linear increase. A similar pattern is observed with regard to mean velocity, with both gases causing a reduction as $S_{g}$ approaches 1.02, after which $\mathrm{CO}_{2}$ continues to cause a reduction whilst $\mathrm{CH}_{4}$ displays a trend of increasing velocity.

Between $S_{g}=1.05$ and 1.02, methane and carbon dioxide both cause a reduction in size and velocity of HMFR as the $\mathrm{CRZ}$ is increasing in size. Although the premixed air and fuel mixture was stoichiometric, the flame was unconfined, and in an environment with a large excess of oxidant. This facilitated a large increase in the volume of the structures when methane was injected, whereas the cooling effect of the carbon dioxide actually reduced structure volume.

There are four mechanisms that may result in flame flashback [7, 9-11]; upstream flame propagation of coherent structures and boundary layer flame propagation in 

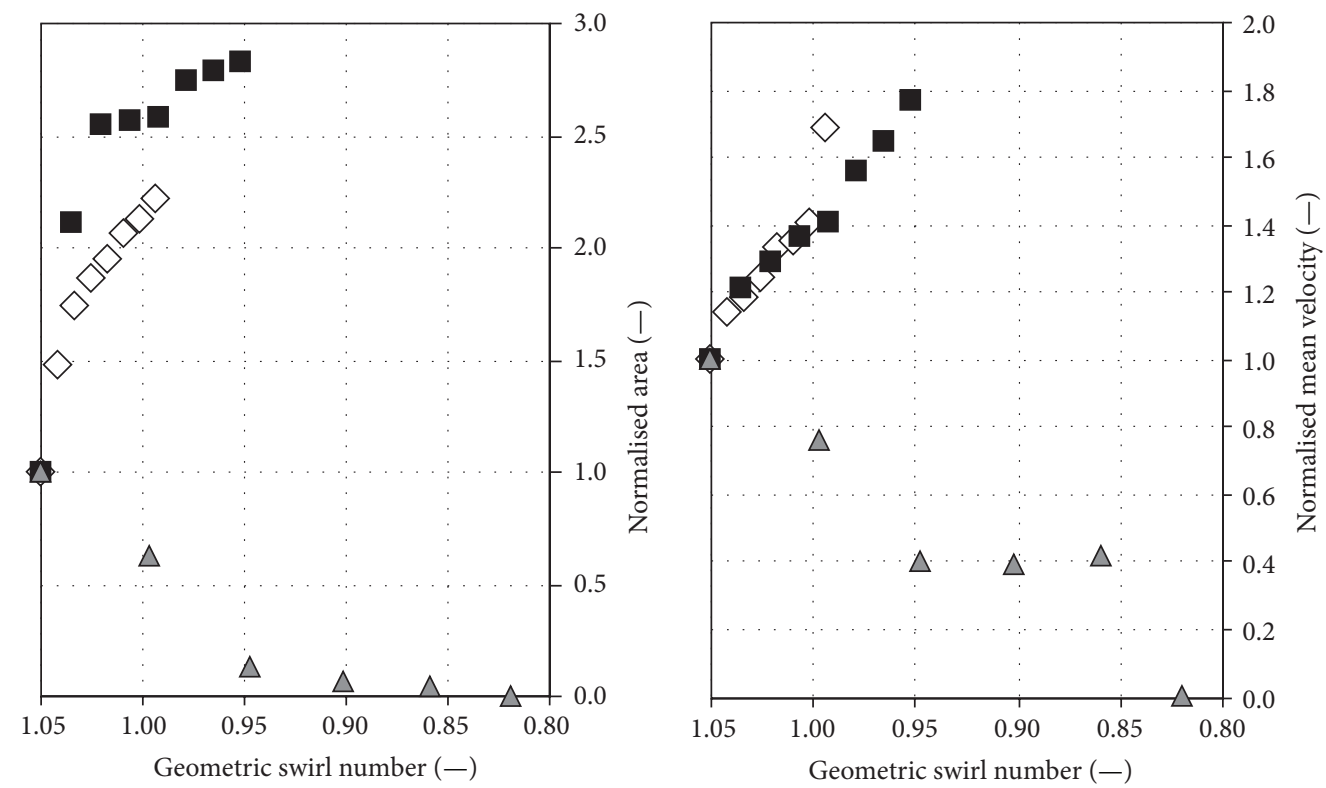

$\diamond$ Carbon dioxide

- Methane

$\diamond$ Carbon dioxide

$\Delta$ Air

- Methane

$\Delta$ Air

(a)

(b)

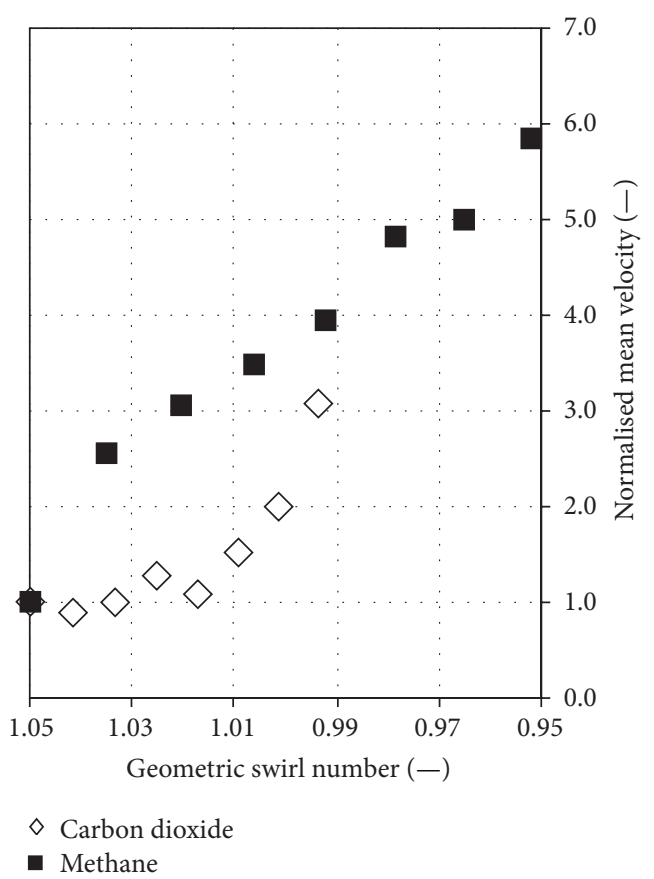

(c)

FIGURE 11: The normalised effect of the injection of carbon dioxide, methane, and air on (a) cross sectional area and (b) mean velocity of the entire CRZ and (c) mean velocity of the CRZ centre.

particular are symptomatic of combustion induced vortex breakdown.

Previous studies have demonstrated that CIVB can be the result of the CRZ being "squeezed" by the HMFR, which causes the CRZ to be displaced, upstream, ultimately surrounding a central body in the combustor [12]. An increase in equivalence ratio caused this process to occur. However, depending on burner design, and critically the level of turbulence of the flow, it may also be caused by a reduction in equivalence ratio [25]. 


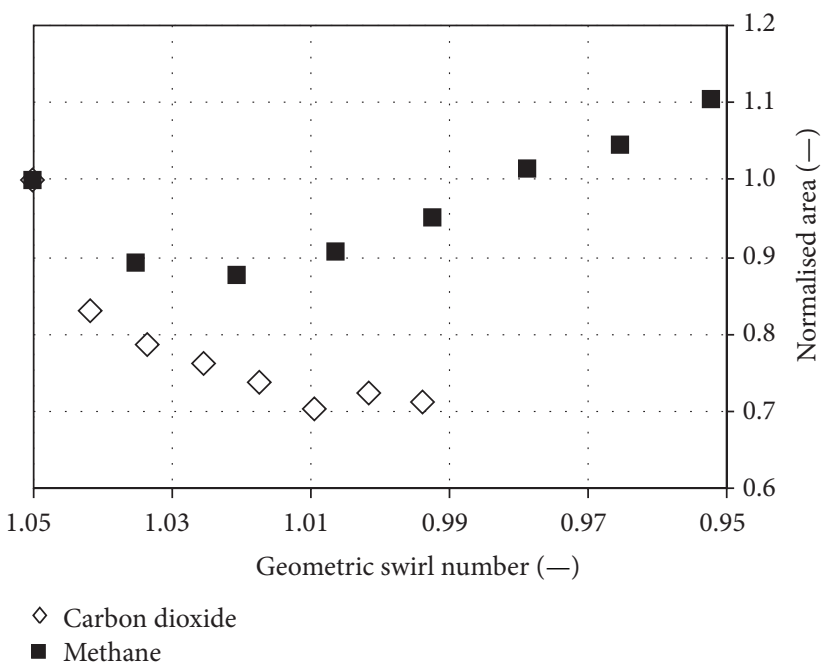

(a)

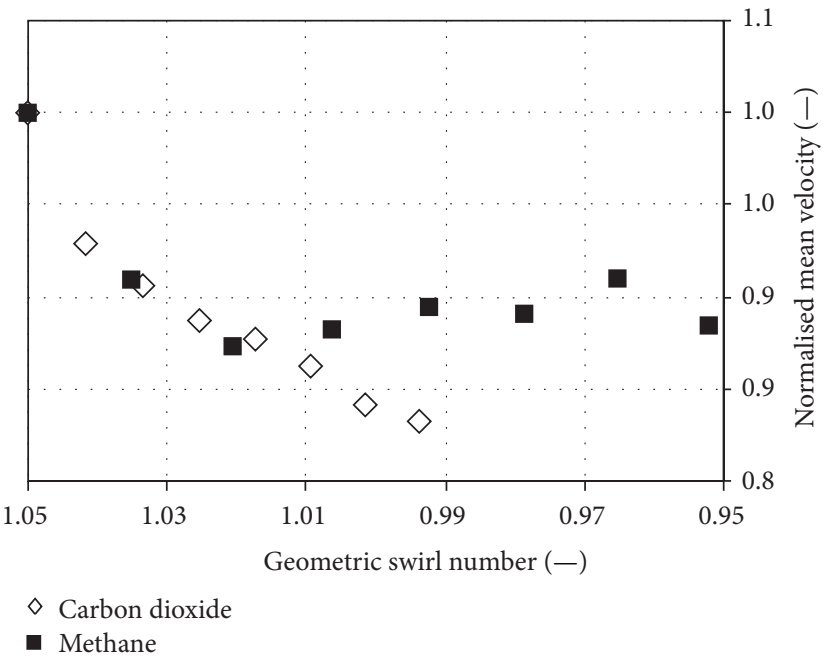

(b)

FIGURE 12: The normalised effect of the injection of carbon dioxide and methane on (a) the cross sectional area and (b) mean velocity of the HMFR.

Regardless of equivalence ratio changes, the interactions between the CRZ and HMFR flow structures are important factors relating to the initiation of CIVB, and this interaction may be altered by the diffusive injection of gases.

For the results taken in these trials the interaction between the CRZ and HMFR is assessed as described in (4), where $R_{M}$ refers to the ratio of total RFM in the CRZ and total PFM in the HMFR; $A_{\mathrm{CRZ}}, A_{\mathrm{HMFR}}, \bar{U}_{\mathrm{CRZ}}$, and $\bar{U}_{\mathrm{HMFR}}$ refer to the cross sectional area and mean axial velocities of the CRZ and HMFR, respectively:

$$
R_{M}=\frac{A_{\mathrm{CRZ}} \cdot \bar{U}_{\mathrm{CRZ}}}{A_{\mathrm{HMFR}} \cdot \bar{U}_{\mathrm{HMFR}}} .
$$

The full size of the HMFR is cropped by the measurement area. However, local changes in the measured area are indicative of global changes. The increasing value of $R_{M}$ as more diffusive fuel is added indicates that the RFM of the CRZ is increasing compared to the PFM of the HMFR, and as a result reducing the propensity of the flame to flashback due to combustion induced vortex breakdown. The normalised effects of carbon dioxide and methane on $R_{M}$ are displayed in Figure 13.

The differing effects of $\mathrm{CO}_{2}$ and $\mathrm{CH}_{4}$ on the $\mathrm{CRZ}$, and in particular the HMFR, compensate for each other to produce a very similar response on the flame structure as a whole. The introduction of methane causes a large initial rise in $R_{M}$ as $S_{g}$ approaches 1.02, at which point the rate of change reduces dramatically. When $1.05<S_{g}<1.02$, there is a strong change in $R_{M}$ as the interaction between the CRZ and HMFR is altered, and with $S_{g}<1.02$, the change in $R_{M}$ becomes less significant as the increased flow rate of methane causes an increase in global flame structure size and velocity.

Carbon dioxide produces a near linear response over the range of results, with the highest value of $R_{M}$ being greater than that of methane.

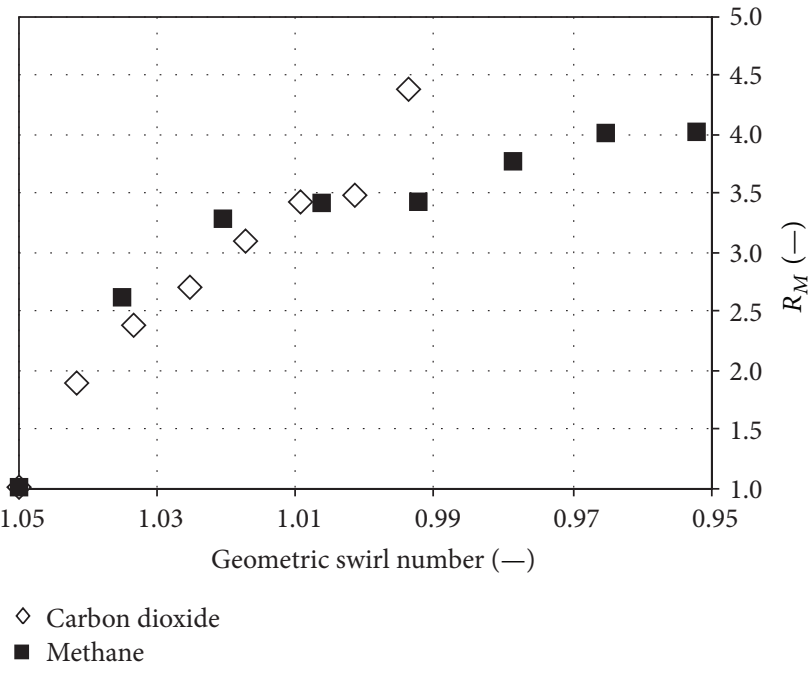

FIGURE 13: Normalised effect of the injection of carbon dioxide and methane on the ratio between RFM and PFM.

\section{Summary and Conclusions}

Experimentation using a generic swirl burner and PIV system demonstrated how the diffusive injection of different gases into a premixed methane flame altered its coherent structures.

The injection of both methane and carbon dioxide resulted in an increase in cross sectional area and velocity of gases within the central recirculation zone whilst maintaining flame stability. For the unconfined flame, diffusive injection of methane increased the burning rates and temperatures within the swirling flows, effecting flame propagation and hot gas expansion. Radial momentum increased whilst axial 
momentum decreased, increasing the volume of the recirculated gases when methane was injected. This leads to an expansion of the recirculation zone near the burner exit and a stable, well-defined boundary between the CRZ and high momentum flow region. The injection of carbon dioxide reduced the burning rate and temperature of gases within the flame, inhibiting flame propagation and reducing the hot gas expansion. Both axial and radial momentums were proportionally reduced, so the increased mass flow rate increased the size and velocity of the recirculation zone.

A differing effect on the high momentum flow region of the flame was seen. When flow rates of the diffusively injected gases produced isothermal geometric swirl numbers above 1.02, the size and velocity of the HMFR decreased, due to $\mathrm{CH}_{4}$ increasing the level of swirl and $\mathrm{CO}_{2}$ decreasing flame temperatures. When methane flow rates resulted in $S_{g}<1.02$, this the cumulative effect of increasing swirl and flame temperature resulted in an increase in HMFR size and velocity.

Despite differing effects of the level of swirl and flame temperature, the actual effect on the RFM of the CRZ compared to the PFM of the HMFR was the same. The expansion of the CRZ compared to the HMFR opposes the changes in flame structure that result in combustion induced vortex breakdown; therefore, the diffusive injection of either $\mathrm{CH}_{4}$ or $\mathrm{CO}_{2}$ could be used as a measure to mitigate CIVB.

Tests were also performed with the diffusive injection of air. Its effect on the flow structures heavily contrasted those of $\mathrm{CO}_{2}$ and $\mathrm{CH}_{4}$, reducing the size and velocity of the CRZ before completely preventing recirculation occurring. Although the overall result would almost certainly reduce the likelihood of CIVB occurring, the destruction of the CRZ would result in an inherently unstable flame that would be highly susceptible to blow off.

\section{Conflict of Interests}

The authors declare that there is no conflict of interests regarding the publication of this paper.

\section{Acknowledgments}

This work was partly funded and supported via the European Union FP7 Project $\mathrm{H}_{2}$ IGCC, and the ERDF funded Low Carbon Research Institute.

\section{References}

[1] A. E. E. Khalil, V. K. Arghode, A. K. Gupta, and S. C. Lee, "Low calorific value fuelled distributed combustion with swirl for gas turbine applications," Applied Energy, vol. 98, pp. 69-78, 2012.

[2] A. Doherty, E. E. Walsh, and K. McDonnell, "The direct use of post-processing wood dust in gas turbines," Journal of Sustainable Bioenergy Systems, vol. 2, no. 3, pp. 60-64, 2012.

[3] C. Eichler, G. Baumgartner, and T. Sattelmayer, "Experimental investigation of turbulent boundary layer flashback limits for premixed hydrogen-air flames confined in ducts," Journal of Engineering for Gas Turbines and Power, vol. 134, no. 1, Article ID 011502, 2012.
[4] N. Syred, M. Abdulsada, A. Griffiths, T. O’Doherty, and P. Bowen, "The effect of hydrogen containing fuel blends upon flashback in swirl burners," Applied Energy, vol. 89, no. 1, pp. 106-110, 2012.

[5] J. Bower, D. Abbott, and S. James, "The impact of natural gas composition variations on the operation of gas turbines for power generation," in Proceedings of the 6th International Conference on Future Gas Turbine Technology, Brussels, Belguim, 2012.

[6] M. Subramanya and A. Choudhuri, "Investigation of combustion instability effects on the flame characteristics of fuel blends," in Proceedings of the 5th International Energy Conversion Engineering Conference (IECEC '07), pp. 817-830, June 2007.

[7] T. Lieuwen, V. McDonell, D. Santavicca, and T. Sattelmayer, "Burner development and operability issues associated with steady flowing syngas fired combustors," Combustion Science and Technology, vol. 180, no. 6, pp. 1169-1192, 2008.

[8] J. D. Thornton, B. T. Chorpening, T. G. Sidwell, P. A. Strakey, E. D. Huckaby, and K. J. Benson, "Flashback detection sensor for hydrogen augmented natural gas combustion," in Proceedings of the ASME Conference, pp. 739-746, May 2007.

[9] J. Fritz, M. Kröner, and T. Sattelmayer, "Flashback in a swirl burner with cylindrical premixing zone," Journal of Engineering for Gas Turbines and Power, vol. 126, no. 2, pp. 276-283, 2004.

[10] M. Kroner, "Flashback limits for combustion induced vortex breakdown in a swirl burner," ASME Paper GT-2002-30075, ASME Turbo Expo, Amsterdam, The Netherlands, 2002.

[11] M. Kröner, J. Fritz, and T. Sattelmayer, "Flashback limits for combustion induced vortex breakdown in a swirl burner," Journal of Engineering for Gas Turbines and Power, vol. 125, no. 3, pp. 693-700, 2003.

[12] B. Dam, G. Corona, M. Hayder, and A. Choudhuri, "Effects of syngas composition on combustion induced vortex breakdown (CIVB) flashback in a swirl stabilized combustor," Fuel, vol. 90, no. 11, pp. 3274-3284, 2011.

[13] B. Dam, N. Love, and A. Choudhuri, "Flashback propensity of syngas fuels," Fuel, vol. 90, no. 2, pp. 618-625, 2011.

[14] C. Eichler and T. Sattelmayer, "Experiments on flame flashback in a quasi-2D turbulent wall boundary layer for premixed methane-hydrogen-air mixtures," Journal of Engineering for Gas Turbines and Power, vol. 133, no. 1, Article ID 011503, 2011.

[15] C. Eichler and T. Sattelmayer, "Premixed flame flashback in wall boundary layers studied by long-distance micro-PIV," Experiments in Fluids, vol. 52, no. 2, pp. 347-360, 2012.

[16] A. Valera-Medina, Coherent structures and their effects on processes occurring in swirl combustors [Ph.D. thesis], Cardiff University, 2009.

[17] A. Valera-Medina, N. Syred, and A. Griffiths, "Visualisation of isothermal large coherent structures in a swirl burner," Combustion and Flame, vol. 156, no. 9, pp. 1723-1734, 2009.

[18] A. Valera-Medina, N. Syred, P. Bowen, and A. Crayford, "Studies of swirl burner characteristics, flame lengths and relative pressure amplitudes," Journal of Fluids Engineering, vol. 133, no. 10, Article ID 101302, 2011.

[19] A. Valera-Medina, N. Syred, P. Kay, and A. Griffiths, "Central recirculation zone analysis in an unconfined tangential swirl burner with varying degrees of premixing," Experiments in Fluids, vol. 50, no. 6, pp. 1611-1623, 2011.

[20] A. Valera Medina, N. Syred, and P. Bowen, "Central recirculation zone analysis using a confined swirl burner for terrestrial energy," Journal AIAA Propulsion and Power, vol. 29, no. 1, pp. 195-204, 2013. 
[21] M. Stöhr, I. Boxx, C. D. Carter, and W. Meier, "Experimental study of vortex-flame interaction in a gas turbine model combustor," Combustion and Flame, vol. 159, no. 8, pp. 26362649, 2012.

[22] K. S. Kedia and A. F. Ghoniem, "Mechanisms of stabilization and blowoff of a premixed flame downstream of a heatconducting perforated plate," Combustion and Flame, vol. 159, no. 3, pp. 1055-1069, 2012.

[23] M. Kröner, T. Sattelmayer, J. Fritz, F. Kiesewetter, and C. Hirsch, "Flame propagation in swirling flows: effect of local extinction on the combustion induced vortex breakdown," Combustion Science and Technology, vol. 179, no. 7, pp. 1385-1416, 2007.

[24] G. Blesinger, R. Koch, and H. J. Bauer, "Influence of flow field scaling on flashback of swirl flames," Experimental Thermal and Fluid Science, vol. 34, no. 3, pp. 290-298, 2010.

[25] J. Lewis, R. Marsh, A. Valera-Medina, S. Morris, and H. Baej, "The use of $\mathrm{CO}_{2}$ to improve stability and emissions of an IGCC combustor," in ASME Turbo Expo 2014: Turbine Technical Conference and Exposition, Dusseldorf, Germany, June 2014.

[26] J. Warnatz, U. Maas, and R. Dibble, Combustion, Springer, Berlin, Germany, 1999.

[27] M. C. Lee, S. B. Seo, J. Yoon, M. Kim, and Y. Yoon, "Experimental study on the effect of $\mathrm{N}_{2}, \mathrm{CO}_{2}$, and steam dilution on the combustion performance of $\mathrm{H}_{2}$ and $\mathrm{CO}$ synthetic gas in an industrial gas turbine," Fuel, vol. 102, pp. 431-438, 2012.

[28] J. Park, D. S. Bae, M. S. Cha et al., "Flame characteristics in $\mathrm{H}_{2} / \mathrm{CO}$ synthetic gas diffusion flames diluted with $\mathrm{CO}_{2}$ : effects of radiative heat loss and mixture composition," International Journal of Hydrogen Energy, vol. 33, no. 23, pp. 7256-7264, 2008.

[29] A. Konnov, I. Dyakov, and J. Ruyck, "Nitric oxide formation in premixed flames of $\mathrm{H}_{2}+\mathrm{CO}+\mathrm{CO}_{2}$ and air," Proceedings of the Combustion Institute, vol. 295, pp. 2171-2177, 2002.

[30] J. Natarajan, T. Lieuwen, and J. Seitzman, "Laminar flame speeds of $\mathrm{H}_{2} / \mathrm{CO}$ mixtures: Effect of $\mathrm{CO}_{2}$ dilution, preheat temperature, and pressure," Combustion and Flame, vol. 151, no. 1-2, pp. 104-119, 2007.

[31] H. J. Burbano, J. Pareja, and A. A. Amell, "Laminar burning velocities and flame stability analysis of $\mathrm{H}_{2} / \mathrm{CO} /$ air mixtures with dilution of $\mathrm{N}_{2}$ and $\mathrm{CO}_{2}$," International Journal of Hydrogen Energy, vol. 36, no. 4, pp. 3232-3242, 2011.

[32] N. Syred and J. M. Beér, "Combustion in swirling flows: a review," Combustion and Flame, vol. 23, no. 2, pp. 143-201, 1974.

[33] C. T. Bowman, M. Frenklach, W. C. Gardiner, and G. P. Smith, "The "GRIMech 3.0" chemical kinetic mechanism," 1999, http://www.me.berkeley.edu/gri-mech/.

[34] S. de Persis, F. Foucher, L. Pillier, V. Osorio, and I. Gökalp, "Effects of $\mathrm{O}_{2}$ enrichment and $\mathrm{CO}_{2}$ dilution on laminar methane flames," Energy, vol. 55, pp. 1055-1066, 2013. 

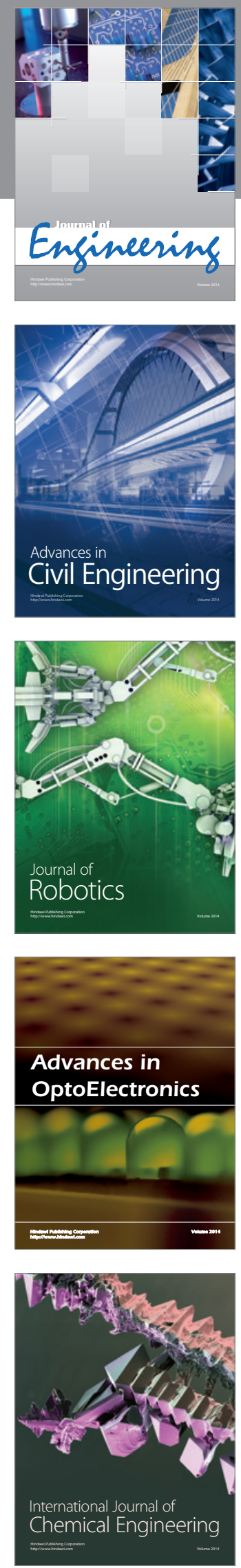

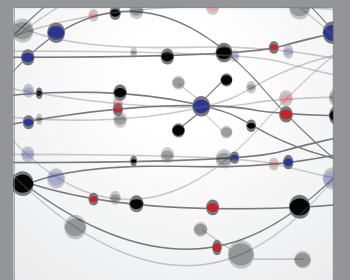

The Scientific World Journal
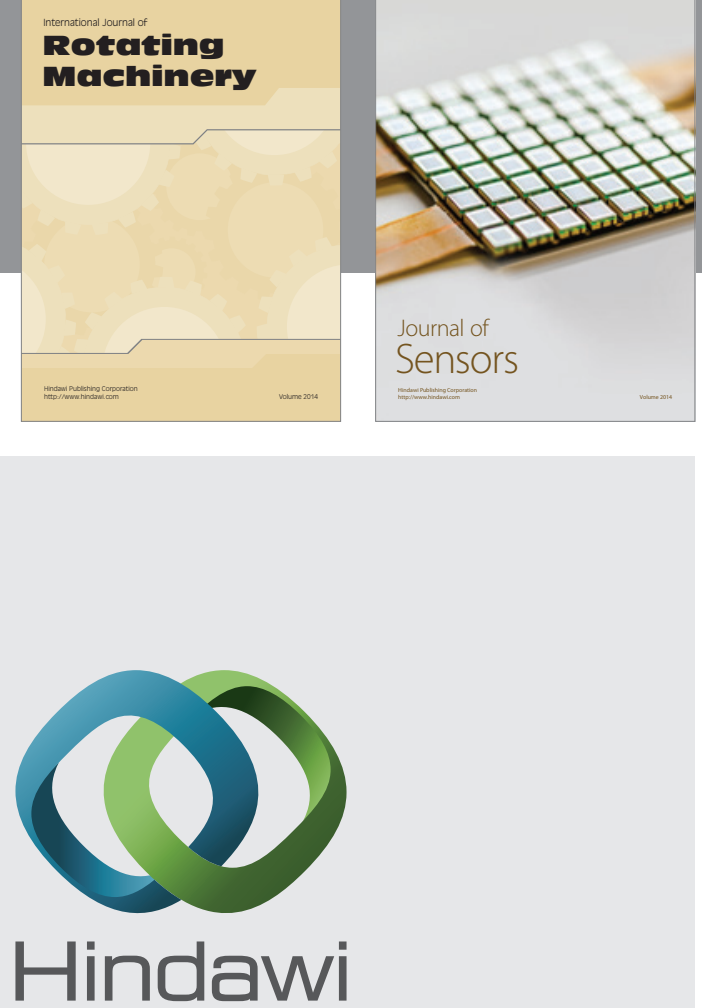

Submit your manuscripts at http://www.hindawi.com
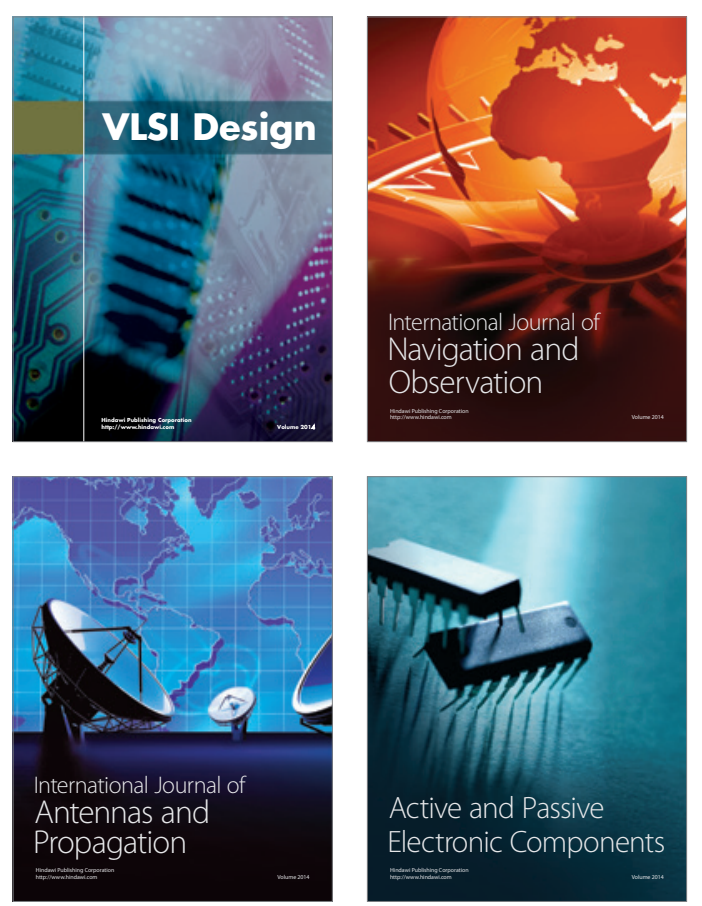
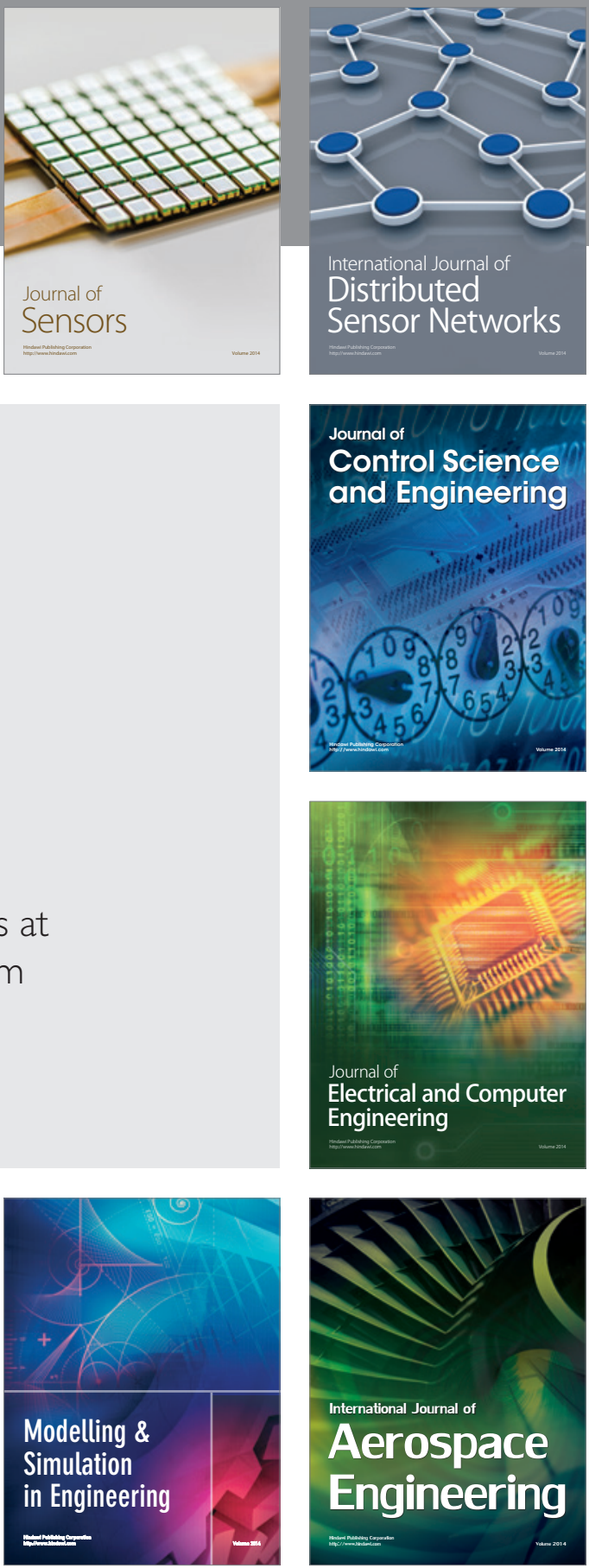

Journal of

Control Science

and Engineering
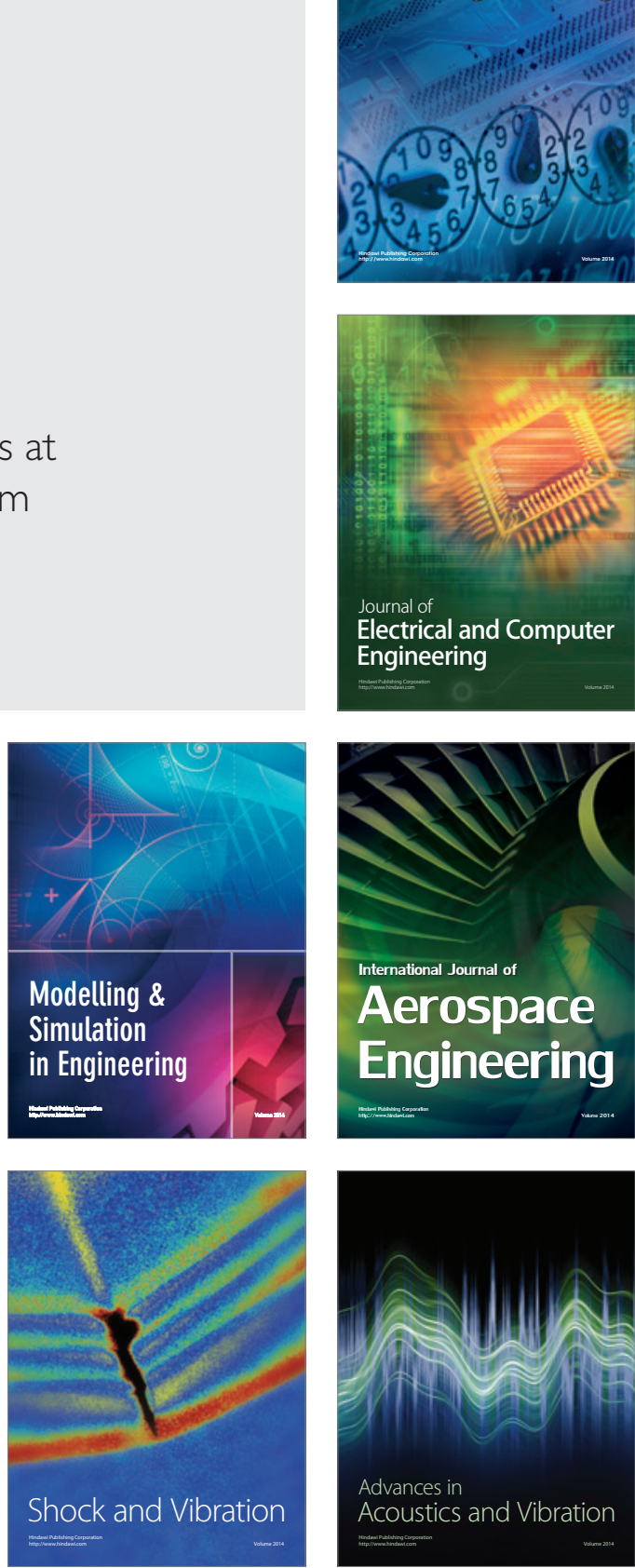